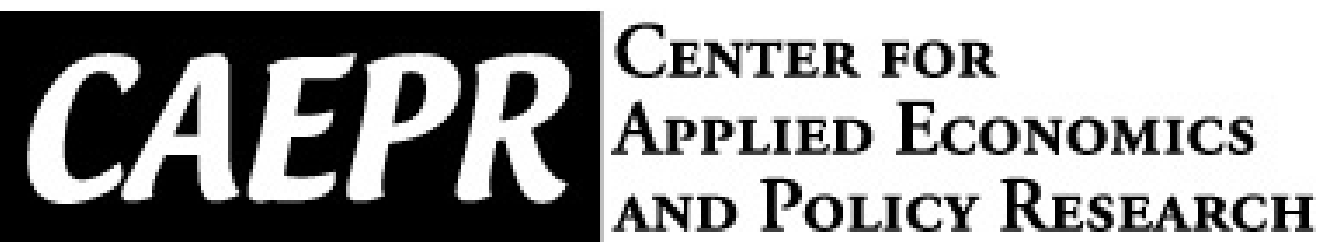

CAEPR Working Paper

\#019-2009

\title{
ASYMPTOTIC DISTRIBUTION-FREE DIAGNOSTIC TESTS FOR HETEROSKEDASTIC TIME SERIES MODELS
}

\author{
J. Carlos Escanciano \\ Indiana University \\ September 10, 2009
}

This paper can be downloaded without charge from the Social Science Research Network electronic library at: http://ssrn.com/abstract=1492177.

The Center for Applied Economics and Policy Research resides in the Department of Economics at Indiana University Bloomington. CAEPR can be found on the Internet at:

http://www.indiana.edu/ caepr. CAEPR can be reached via email at caepr@indiana.edu or via phone at 812-855-4050.

(C2008 by NAME. All rights reserved. Short sections of text, not to exceed two paragraphs, may be quoted without explicit permission provided that full credit, including $\odot$ notice, is given to the source. 


\title{
ASYMPTOTIC DISTRIBUTION-FREE DIAGNOSTIC TESTS FOR HETEROSKEDASTIC TIME SERIES MODELS*
}

\author{
J. CARlos Escanciano ${ }^{\dagger}$ \\ Indiana University
}

September 10, 2009

\begin{abstract}
This article investigates model checks for a class of possibly nonlinear heteroskedastic time series models, including but not restricted to ARMA-GARCH models. We propose omnibus tests based on functionals of certain weighted standardized residual empirical processes. The new tests are asymptotically distribution-free, suitable when the conditioning set is infinitedimensional, and consistent against a class of Pitman's local alternatives converging at the parametric rate $n^{-1 / 2}$, with $n$ the sample size. A Monte Carlo study shows that the simulated level of the proposed tests is close to the asymptotic level already for moderate sample sizes and that tests have a satisfactory power performance. Finally, we illustrate our methodology with an application to the well-known S\&P 500 daily stock index. The paper also contains an asymptotic uniform expansion for weighted residual empirical processes when initial conditions are considered, a result of independent interest.
\end{abstract}

Keywords and Phrases: Time series models; model specification; ARMA-GARCH models; S\&P 500.

Jel Classification: C12; C52.

\footnotetext{
*Research funded by the Spanish Plan Nacional de I+D+I, reference number SEJ2007-62908 and by the Spanish Ministerio de Educación y Ciencia, reference number SEJ2005-07657/ECON.

$\dagger$ Department of Economics, Indiana University; 100 S. Woodlawn, Wylie Hall; Bloomington, IN 47405-7104; USA, e-mail: jescanci@indiana.edu. I would like to thank Miguel A. Delgado, Oliver Linton, Carlos Velasco and two anonymous referees for helpful comments. I also thank Wenceslao González-Manteiga for pointing out an important reference. All errors are mine.
} 


\section{INTRODUCTION}

The correct specification of conditional mean and variance models is of major interest in empirical finance and economics. Model-based economic theories in dynamic contexts, such as asset pricing, portfolio choice and market risk management, as well as many inference procedures proposed in the econometrics literature, such as the consistency of the Quasi-Maximum Likelihood Estimator (QMLE), depend crucially on the correct joint specification of the conditional mean and variance. Despite the significant empirical and theoretical importance of this testing problem, there exist rather few joint specification tests available in the literature. Furthermore, most of the existing tests have model-dependent asymptotic distributions and may require bootstrap methods that are difficult to implement in standard econometric packages. The aim of this paper is then to construct simple asymptotic distribution-free (ADF) joint specification tests, especially convenient for financial and economic applications. The new tests are based on certain weighted standardized residual empirical processes with weights equal to proper transformations of the conditioning variables. The weights are chosen orthogonal to model's scores, so ADF omnibus tests are obtained, with the added property of being flexible to tailor the power against desired directions.

To be more precise, let $\left\{Y_{t}\right\}_{t \in \mathbb{Z}}$ be a strictly stationary and ergodic time series process defined on the probability space $(\Omega, \mathcal{F}, P)$. A large body of the time series modeling literature, both theoretical and empirical, has considered the model

$$
Y_{t}=f\left(I_{t-1}, \theta_{0}\right)+h\left(I_{t-1}, \theta_{0}\right) u_{t}, \quad t \in \mathbb{Z}
$$

where $I_{t-1}=\left(Y_{t-1}, u_{t-1}, Y_{t-2}, u_{t-2}, \ldots\right)^{\prime}$ is the conditioning set at time $t-1$, and $f\left(I_{t-1}, \theta_{0}\right)$ and $h^{2}\left(I_{t-1}, \theta_{0}\right)$ are parametric specifications for $f(y):=E\left[Y_{t} \mid I_{t-1}=y\right]$ almost surely (a.s.) and $h^{2}(y):=\operatorname{Var}\left[Y_{t} \mid I_{t-1}=y\right]$ a.s. In (1), $\theta_{0}$ is an unknown parameter in an Euclidean space,

$\theta_{0} \in \Theta \subset \mathbb{R}^{p}$, and $\left\{u_{t}\right\}$ is a sequence of independent and identically distributed (iid) disturbances, with unknown cumulative distribution function (cdf) $F_{u}$. Throughout the paper we assume that $u_{t}$ is independent of $I_{t-1}$, for all $t \in \mathbb{Z}$. The specification (1) covers the well-known linear ARMA-GARCH models, as well as nonlinear conditional mean and variance models, see, e.g., Fan and Yao (2003). Our theory is valid for the general heteroskedastic time series model described in (1).

We aim to test under this set-up for

$$
H_{0}: f\left(I_{t-1}\right)=f\left(I_{t-1}, \theta_{0}\right) \text { and } h\left(I_{t-1}\right)=h\left(I_{t-1}, \theta_{0}\right) \text { a.s. for some } \theta_{0} \in \Theta \text {, }
$$

against nonparametric or parametric alternatives given by the negation of $H_{0}$. This testing problem has important implications in econometrics practice and, in particular, in financial econometrics 
modeling where parametric models such as (1) are commonly used. An example of application is to the Value at Risk (VaR) methodology in market risk management, see Tsay (2005). The VaR at level $\alpha$ in model (1) is given by $\operatorname{VaR}_{t}(\alpha)=f\left(I_{t-1}, \theta_{0}\right)+h\left(I_{t-1}, \theta_{0}\right) F_{u}^{-1}(\alpha)$, where $F_{u}^{-1}(\alpha)$ is the standardized error's quantile function evaluated at $\alpha$. Clearly, the functional forms for $f$ and $h$ play a crucial role in determining the $\operatorname{VaR}_{t}(\alpha)$, whereas $\theta_{0}$ and $F_{u}^{-1}(\alpha)$ are nuisance parameters that can be estimated by many $\sqrt{n}$-consistent estimators, e.g. QMLE for $\theta_{0}$ and quantile estimators for $F_{u}^{-1}(\alpha)$, see e.g. Koenker and Zhao (1996).

More generally, model specification within the class (1) is important mainly because a lack of fit in the postulated conditional mean and/or variance can lead to misleading conclusions in statistical inferences, and to suboptimal point forecasts. Therefore, in order to prevent wrong conclusions, every statistical inference that is based on the model $\mathcal{M}=\left\{f(\cdot, \theta), h(\cdot, \theta): \theta \in \Theta \subset \mathbb{R}^{p}\right\}$ should be accompanied by a proper joint diagnostic tests, i.e., a test for $H_{0}$.

There is a vast amount of literature on testing the specification of parametric dynamic conditional mean models, see Escanciano (2006) and references therein. On the contrary, the literature on joint model checks for the conditional mean and variance functions is rather scarce. Classical diagnostic tools for testing $H_{0}$ are the Portmanteau tests initially proposed by Box and Pierce (1970) and Ljung and Box (1978), and subsequently extended to some conditional variance models by Li and Mak (1994), see also Lundbergh and Teräsvirta (2002) and Hidalgo and Zaffaroni (2007). Tests based on usual correlation or autocorrelation measures of errors (centered squared errors) are not consistent in any misspecified model that lead to uncorrelated errors (centered squared errors), increasing the Type II error probability. Ngatchou-Wandji (2005) proposed using $\chi^{2}$-discrepancy measures for testing $H_{0}$, whereas Gao and King (2004) have extended the initial smoothed-based approach of Härdle and Mammen (1993) to tests for $H_{0}$.

An important limitation of all the aforementioned articles, with the exception of Hidalgo and Zaffaroni (2007), is that they consider a finite-dimensional information set $I_{t-1}$, and hence, they are not suitable for testing $H_{0}$ here. Moreover, even for the case in which the information set is of finite dimension, $d$ say, most of the proposed tests lead to a poor power performance when $d$ is large or moderate, due to the so-called "curse of dimensionality" problem. Escanciano (2008) overcomes the latter problem using a generalized spectral approach, but resorting to the assistance of a bootstrap approximation that is difficult to implement in standard econometric packages. Alternatively, Hong and Lee (2003) considered a smoothed approach based on the generalized spectral density which requires a bandwidth choice, without much guidance for its choice for testing purposes.

This article deviates from previous works by proposing a large class of simple ADF tests for $H_{0}$ that are robust to the dimension in the information set $I_{t-1}$. The rationale for our tests follows from 
the asymptotic properties of the weighted empirical processes of standardized residuals

$$
R_{n, \widehat{g}}^{1}(x):=\frac{1}{\sqrt{n}} \sum_{t=1}^{n} \widehat{g}_{c}\left(\widehat{I}_{t-1}, \theta_{n}\right) 1\left(\widehat{u}_{t} \leq x\right),
$$

where $\widehat{g}_{c}\left(\widehat{I}_{t-1}, \theta_{n}\right)$ is an estimator for $g_{c}\left(I_{t-1}, \theta_{0}\right)$, a suitable transformation of the conditioning set which will be specified later on, $\widehat{u}_{t}$ are standardized residuals obtained from (1) and computed as

$$
\widehat{u}_{t} \equiv \widehat{u}_{t}\left(\theta_{n}\right)=\frac{Y_{t}-f\left(\widehat{I}_{t-1}, \theta_{n}\right)}{h\left(\widehat{I}_{t-1}, \theta_{n}\right)}, \quad 1 \leq t \leq n,
$$

where $\theta_{n}$ is a $\sqrt{n}$-consistent estimators for $\theta_{0}$, e.g. the QMLE, and $\widehat{I}_{t-1}$ is the information set observed at time $t-1$ that contains $\left(Y_{t-1}, Y_{t-2}, \ldots, Y_{0}\right)$ and that may contain some other initial values; see Section 3.

As we shall show, the asymptotic behaviour of the process $R_{n, \widehat{g}}^{1}$ will depend on whether $H_{0}$ holds or not. This fact will allow us to base omnibus tests on suitable functionals of $R_{n, \widehat{g}}^{1}$. We choose the weights $\widehat{g}_{c}\left(\widehat{I}_{t-1}, \theta_{n}\right)$ in $(2)$ in such a way that simple ADF tests are obtained, avoiding much of the burden of bootstrap approximations or complicated martingale transforms.

Our tests can be seen as generalizations of the tests proposed by Wooldridge (1990). This author considers tests based on e.g.

$$
\frac{1}{\sqrt{n}} \sum_{t=1}^{n} \widehat{g}_{c}\left(I_{t-1}, \theta_{n}\right) \widehat{u}_{t}, \quad \text { and } \quad \frac{1}{\sqrt{n}} \sum_{t=1}^{n} \widehat{g}_{c}\left(I_{t-1}, \theta_{n}\right) \widehat{u}_{t}^{2} .
$$

Note that both quantities in (3) are empirical integrals of $R_{n, \widehat{g}}^{1}$. In particular,

$$
\int_{\mathbb{R}} x R_{n, \widehat{g}}^{1}(d x)=\frac{1}{\sqrt{n}} \sum_{t=1}^{n} \widehat{g}_{c}\left(I_{t-1}, \theta_{n}\right) \widehat{u}_{t},
$$

so the sample means in (3) contain less "information" than the process $R_{n, \widehat{g}}^{1}$ itself, resulting in generally less powerful testing procedures, see Section 2.2 for discussion.

Weighted residual empirical processes have been used in the literature for different testing problems than $H_{0}$. Koul and Ling (2006) proposed goodness of fit tests for the error distribution in (1) assuming the conditional mean and variance are correctly specified. Thus, our tests here can be used as a pre-test in Koul and Ling's (2006) goodness of fit test. Also, we stress that in practice the information set $I_{t-1}$ is not observable and must estimated by $\widehat{I}_{t-1}$. Koul and Ling (2006) did not take into account this estimation of initial values, and unfortunately this makes the application of their results difficult; see the discussion in the next section. Therefore, in order to carry out our program we need to extend a weak convergence result in Koul and Ling (2006, Lemma 4.1), see also Lemma A.6 in Bai and Ng (2001), to allow for an estimated conditioning set. This extension is not trivial and is of independent interest. 
We also note that after the first version of the paper was written, it came to our attention ${ }^{1}$ a recent paper by Stute, Xu and Zhu (2008) using weighted residual processes for testing the correct specification of a homokedastic regression in a iid setup. These authors developed a principal components decomposition that can be also used in the present framework. Details are omitted to save space. Nevertheless, the testing framework in Stute et al. (2008) is different from ours and uses different tools.

The remainder of this paper is organized as follows. In Section 2 we study the asymptotic theory for a class of weighted residual empirical processes and introduce the tests. In Section 3 we illustrate the applicability of our methods and show that our assumptions are naturally satisfied by the class of ARMA-GARCH models. Section 4 provides evidence of the good finite-sample performance of the proposed tests and apply the new methods to the S\&P 500 daily stock index. Section 5 concludes and discusses future extensions. In the Appendix we establish an asymptotic uniform expansion for a weighted residual empirical processes when initial conditions are considered, a result of independent interest. Mathematical proofs are gathered in the Appendix.

\section{JOINT MODEL CHECKS FOR MEAN-VARIANCE MODELS}

\subsection{Asymptotic null distribution}

This section introduces omnibus ADF tests for $H_{0}$. For the sake of exposition we shall first consider the limit behaviour of the process

$$
R_{n, g}^{1}(x):=\frac{1}{\sqrt{n}} \sum_{t=1}^{n} g_{c}\left(\widehat{I}_{t-1}, \theta_{n}\right) 1\left(\widehat{u}_{t} \leq x\right)
$$

for a centered weight $g_{c}\left(I_{t-1}, \theta\right)=g\left(I_{t-1}, \theta\right)-\bar{g}(\theta), \bar{g}(\theta)=n^{-1} \sum_{t=1}^{n} g\left(I_{t-1}, \theta\right)$, where $g\left(I_{t-1}, \theta\right)$ is a generic real-valued measurable transformation of $I_{t-1}$ that is smooth in $\theta$ (cf. A5). Henceforth, the subindexes $c$ and $e$ stand for sample and population centering, respectively, e.g. $g_{e}\left(I_{t-1}, \theta\right):=$ $g\left(I_{t-1}, \theta\right)-E\left[g\left(I_{t-1}, \theta\right)\right]$.

We introduce some regularity conditions. For any differentiable function $g$ on $\Theta$, let $\dot{g}$ denote its differential. Throughout, $A^{\prime}$ denotes transposition of the matrix $A$ and $\Theta_{0}$ denotes a small convex neighborhood of $\theta_{0}$. For positive finite constants $b>0$ and $c>0$ define the set $\mathcal{Z}_{b, c}:=\left\{\left(z_{1}, z_{2}\right)^{\prime} \in\right.$ $\left.\mathbb{R}^{2}:\left|z_{1}\right|+\left|z_{2}\right| \leq b, z_{1} \geq-1+c\right\}$.

\section{Assumption A1:}

$A 1(a):\left\{Y_{t}\right\}_{t \in \mathbb{Z}}$ is a strictly stationary and ergodic process. $\left\{u_{t}\right\}$ is a sequence of (iid) disturbances, with $E\left[\left|u_{t}\right|\right]<\infty$.

\footnotetext{
${ }^{1}$ We thank Wenceslao González-Manteiga for pointing out this reference.
} 
A1(b): $F_{u}$ has an absolutely continuous density $f_{u}$ such that for all $0<b<\infty$ and $0<c<\infty$,

$\sup _{x \in \mathbb{R},\left(z_{1}, z_{2}\right)^{\prime} \in \mathcal{Z}_{b, c}}|x| f_{u}\left(x+x z_{1}+z_{2}\right)<\infty, \quad \sup _{x \in \mathbb{R}, \gamma \geq-1+c}\left|x^{j} f_{u}(x(1+\gamma))-x^{j} f_{u}(x)\right| \leq C \gamma$ for $j=0,1$.

Assumption A2: The functions $f\left(I_{t-1}, \cdot\right)$ and $h\left(I_{t-1}, \cdot\right)$ are twice continuously differentiable with respect to $\theta \in \Theta_{0}$ a.s., with derivatives $\dot{f}_{t}(\theta)$ and $\dot{h}_{t}(\theta)$, respectively, such that

$$
\begin{gathered}
E\left[\sup _{\theta \in \Theta_{0}}\left|\frac{\dot{f}_{t}(\theta)}{h\left(I_{t-1}, \theta_{0}\right)}\right|^{2}\right]<\infty, \quad E\left[\sup _{\theta \in \Theta_{0}}\left|\frac{\dot{h}_{t}(\theta)}{h\left(I_{t-1}, \theta_{0}\right)}\right|^{2}\right]<\infty, \\
n^{-1 / 2} \max _{1 \leq t \leq n} E\left[\sup _{\theta \in \Theta_{0}}\left|\frac{\dot{f}\left(\widehat{I}_{t-1}, \theta\right)}{h\left(I_{t-1}, \theta_{0}\right)}\right|\right]=O(1), \quad n^{-1 / 2} \max _{1 \leq t \leq n} E\left[\sup _{\theta \in \Theta_{0}}\left|\frac{\dot{h}\left(\widehat{I}_{t-1}, \theta\right)}{h\left(I_{t-1}, \theta_{0}\right)}\right|\right]=O(1),
\end{gathered}
$$

and

$$
n^{-1 / 2} \max _{1 \leq t \leq n_{\theta} \sup _{0}}\left|\frac{\dot{f}_{t}(\theta)}{h\left(I_{t-1}, \theta_{0}\right)}\right|=o_{P}(1), \quad n^{-1 / 2} \max _{1 \leq t \leq n_{\theta \in \Theta_{0}}} \sup _{\theta}\left|\frac{\dot{h}_{t}(\theta)}{h\left(I_{t-1}, \theta_{0}\right)}\right|=o_{P}(1) .
$$

In addition, $\min _{t \in \mathbb{Z}} \inf _{\theta \in \Theta_{0}} h\left(I_{t-1}, \theta\right) \geq c>0$ and

$$
\max _{1 \leq t \leq n_{\theta \in \Theta_{0}}}\left|\frac{h\left(I_{t-1}, \theta_{0}\right)}{h\left(\widehat{I}_{t-1}, \theta\right)}\right|=O_{P}(1) .
$$

Assumption A3: The parametric space $\Theta$ is compact in $\mathbb{R}^{p}$. The parameter $\theta_{0}$ belongs to the interior of $\Theta$. Under the null hypothesis there exists a unique $\theta_{0} \in \Theta$ such that $\sqrt{n}\left(\theta_{n}-\theta_{0}\right)=O_{P}(1)$.

Assumption A4: The observed information set available at period $t-1, \widehat{I}_{t-1}$, may contain some assumed initial values and satisfies

$$
\begin{aligned}
& \lim _{n \rightarrow \infty} \sum_{t=1}^{n} E^{1 / 2}\left\{\sup _{\theta \in \Theta_{0}}\left|\frac{f\left(\widehat{I}_{t-1}, \theta\right)-f\left(I_{t-1}, \theta\right)}{h\left(I_{t-1}, \theta_{0}\right)}\right|^{2}\right\}<\infty, \\
& \lim _{n \rightarrow \infty} \sum_{t=1}^{n} E^{1 / 2}\left\{\sup _{\theta \in \Theta_{0}}\left|\frac{h\left(\widehat{I}_{t-1}, \theta\right)-h\left(I_{t-1}, \theta\right)}{h\left(I_{t-1}, \theta_{0}\right)}\right|^{2}\right\}<\infty
\end{aligned}
$$

and

$$
n^{-1 / 2} \sum_{t=1}^{n} E \sup _{\theta \in \Theta_{0}}\left|g\left(\widehat{I}_{t-1}, \theta\right)-g\left(I_{t-1}, \theta\right)\right|=o_{P}(1) .
$$

Assumption A5: The function $g\left(I_{t-1}, \cdot\right)$ satisfies $E\left[\sup _{\theta \in \Theta_{0}}\left|g\left(I_{t-1}, \theta\right)\right|^{4}\right]<\infty$ and is continuously differentiable with respect to $\theta \in \Theta_{0}$ a.s., with derivative $\dot{g}_{t}(\theta)$ such that $E\left[\sup _{\theta \in \Theta_{0}}\left|\dot{g}_{t}(\theta)\right|^{2}\right]<\infty$.

Assumption A1 is a condition on the DGP. As we shall show, Assumption A2 holds for commonly used models such as ARMA-GARCH models. Assumption A3 is satisfied under mild conditions for the nonlinear least squares estimator (or its robust modifications, under further regularity assumptions) or for the QMLE, see Koul (2002, Chapters 5 and 8), Hall and Heyde (1980, Chapter 6), 
Francq and Zakoïan (2004), Straumann (2005) and Robinson and Zaffaroni (2006), to mention but a few. Assumption A4 is a start-up value condition. It ensures that the impact of initial values are asymptotically negligible. This condition easily holds for many time series models; see Francq and Zakoïan (2004) for ARMA-GARCH models. We remark that Lemma 4.1 in Koul and Ling (2006) requires

$$
\max _{1 \leq t \leq n}\left|\frac{h\left(\widehat{I}_{t-1}, \theta_{0}\right)-h\left(I_{t-1}, \theta_{0}\right)}{h\left(I_{t-1}, \theta_{0}\right)}\right|=o_{P}(1), \quad \max _{1 \leq t \leq n}\left|\frac{f\left(\widehat{I}_{t-1}, \theta_{0}\right)-f\left(I_{t-1}, \theta_{0}\right)}{h\left(I_{t-1}, \theta_{0}\right)}\right|=o_{P}(1),
$$

which is difficult to verify and may not hold even for simple models such as ARMA-GARCH. For instance, in a homokedastic (i.e. $h\left(I_{t-1}, \theta_{0}\right)=\sigma$ ) MA(1) model with estimated information set $\widehat{I}_{t-1}=\left\{Y_{t-1}, \ldots, Y_{1}, \widehat{u}_{0}\right\}$, where $\widehat{u}_{0}$ is some assumed value for $u_{0}$, the conditions in A4 here hold whereas if $\left|\theta_{0}\right|<1$,

$$
\max _{1 \leq t \leq n}\left|\frac{f\left(\widehat{I}_{t-1}, \theta_{0}\right)-f\left(I_{t-1}, \theta_{0}\right)}{h\left(I_{t-1}, \theta_{0}\right)}\right|=\frac{1}{\sigma}\left|\widehat{u}_{0}-u_{0}\right|\left|\theta_{0}\right|,
$$

which does not converge to zero in probability. For this reason, we extend Koul and Ling's (2006) Lemma 4.1 in the Appendix below. This extension is not trivial and has applications beyond the present problem. Finally, Assumption A.5 is on the weight function $g$ and can be easily checked.

Define

$$
b\left(x, g, \theta_{0}\right):=E\left[g_{e}\left(I_{t-1}, \theta_{0}\right) \frac{\dot{f}_{t}\left(\theta_{0}\right)}{h\left(I_{t-1}\right)}\right] f_{u}(x)+E\left[g_{e}\left(I_{t-1}, \theta_{0}\right) \frac{\dot{h}_{t}\left(\theta_{0}\right)}{h\left(I_{t-1}\right)}\right] x f_{u}(x) .
$$

It is important for our purposes to note that if the moment conditions

$$
E\left[g_{e}\left(I_{t-1}, \theta_{0}\right) \frac{\dot{f}_{t}\left(\theta_{0}\right)}{h\left(I_{t-1}\right)}\right]=0
$$

and

$$
E\left[g_{e}\left(I_{t-1}, \theta_{0}\right) \frac{\dot{h}_{t}\left(\theta_{0}\right)}{h\left(I_{t-1}\right)}\right]=0,
$$

hold, then $b\left(x, g, \theta_{0}\right) \equiv 0$. We are now in position to establish the limit null distribution of $R_{n, g}^{1}$.

\section{THEOREM 1:}

(i) Under Assumptions A1-A5 and $H_{0}$, uniformly in $x \in \mathbb{R}$,

$$
R_{n, g}^{1}(x)=\frac{1}{\sqrt{n}} \sum_{t=1}^{n} g_{e}\left(I_{t-1}, \theta_{0}\right)\left[1\left(u_{t} \leq x\right)-F_{u}(x)\right]+\sqrt{n}\left(\theta_{n}-\theta_{0}\right)^{\prime} b\left(x, g, \theta_{0}\right)+o_{P}(1) .
$$

(ii) If in addition, (4) and (5) hold, then, uniformly in $x \in \mathbb{R}$,

$$
R_{n, g}^{1}(x)=\frac{1}{\sqrt{n}} \sum_{t=1}^{n} g_{e}\left(I_{t-1}, \theta_{0}\right)\left[1\left(u_{t} \leq x\right)-F_{u}(x)\right]+o_{P}(1) .
$$

A corollary of Theorem 1(ii) is that, provided the moment conditions (4) and (5) hold, the null limit distribution of tests based on $R_{n, g}^{1}$ will be ADF (cf. Corollary 1). A natural question is then how 
we construct weights satisfying (4) and (5). We address this problem in the next lines. To simplify notation write $a_{1, t}(\theta)=\dot{f}_{t}(\theta) / h\left(I_{t-1}, \theta\right), a_{2, t}(\theta)=\dot{h}_{t}(\theta) / h\left(I_{t-1}, \theta\right)$ and

$$
X_{t}(\theta)=\left(1, a_{1, t}^{\prime}(\theta), a_{2, t}^{\prime}(\theta)\right)^{\prime} \quad \theta \in \Theta, t=1, \ldots, n .
$$

Suppose we consider an initial weight $g_{0}\left(I_{t-1}\right)$. Then, we proceed by taking as $g_{c}\left(\widehat{I}_{t-1}, \theta_{n}\right)$ the residuals from the least squares regression (provided no exact collinearity exists, otherwise remove the necessary regressors) in

$$
g_{0}\left(\widehat{I}_{t-1}\right)=\gamma^{\prime} \widehat{X}_{t}\left(\theta_{n}\right)+\xi_{t} \quad t=1, \ldots, n,
$$

where $\widehat{X}_{t}\left(\theta_{n}\right)$ uses $\widehat{I}_{t-1}$ instead of $I_{t-1}$. The initial weight function $g_{0}$ is up-to the econometrician and gives flexibility to direct the power against desired directions; see the local power analysis in Section 2.1. The least squares estimator in (6) is

$$
\widehat{\gamma}_{n}\left(\theta_{n}\right)=\left(\sum_{t=1}^{n} \widehat{X}_{t}\left(\theta_{n}\right) \widehat{X}_{t}^{\prime}\left(\theta_{n}\right)\right)^{-1}\left(\sum_{t=1}^{n} \widehat{X}_{t}\left(\theta_{n}\right) g_{0}\left(\widehat{I}_{t-1}\right)\right) .
$$

The estimator $\widehat{\gamma}_{n}\left(\theta_{n}\right)$ estimates $\gamma \equiv \gamma\left(\theta_{0}\right)=\left(E\left[X_{t}\left(\theta_{0}\right) X_{t}^{\prime}\left(\theta_{0}\right)\right]\right)^{-1} E\left[X_{t}\left(\theta_{0}\right) g_{0}\left(I_{t-1}\right)\right]$ and the weight

$$
g\left(I_{t-1}, \theta_{0}\right)=g_{0}\left(I_{t-1}\right)-\gamma^{\prime}\left(\theta_{0}\right) X_{t}\left(\theta_{0}\right)
$$

satisfies (4), (5) and has zero mean, by construction. The function

$$
\widehat{g}\left(\widehat{I}_{t-1}, \theta_{n}\right)=g_{0}\left(\widehat{I}_{t-1}\right)-\widehat{\gamma}_{n}^{\prime}\left(\theta_{n}\right) \widehat{X}_{t}\left(\theta_{n}\right)
$$

estimates $g\left(I_{t-1}, \theta_{0}\right)$ in (7). Finally, our tests are functionals of the weighted residual empirical process

$$
R_{n, \widehat{g}}^{1}(x)=\frac{1}{\sqrt{n}} \sum_{t=1}^{n} \widehat{g}\left(\widehat{I}_{t-1}, \theta_{n}\right) 1\left(\widehat{u}_{t} \leq x\right) .
$$

We introduce regularity conditions that are sufficient conditions for A2 for the present choice of $\widehat{g}\left(\widehat{I}_{t-1}, \theta_{n}\right)$.

Assumption A6: The functions $f\left(I_{t-1}, \cdot\right)$ and $h\left(I_{t-1}, \cdot\right)$ are twice continuously differentiable with respect to $\theta \in \Theta_{0}$ a.s., with derivatives $\dot{f}_{t}(\theta)$ and $\left.\dot{h}_{t}(\theta)\right)$ such that

A6(a): $E\left[\sup _{\theta \in \Theta_{0}}\left|X_{t}(\theta)\right|^{4}\right]<\infty$ and $I(\theta)=E\left[X_{t}(\theta) X_{t}^{\prime}(\theta)\right]$ is positive definite on $\Theta_{0}$.

A6(b): The function $X_{t}(\theta)$ is continuously differentiable with respect to $\theta \in \Theta_{0}$ a.s., with derivative $\dot{X}_{t}(\theta)$ such that $E\left[\sup _{\theta \in \Theta_{0}}\left|\dot{X}_{t}(\theta)\right|^{2}\right]<\infty$.

$\mathrm{A} 6$ (c): $E\left[g_{0}^{4}\left(I_{t-1}\right)\right]<\infty$ and $n^{-1 / 2} \sum_{t=1}^{n}\left|g_{0}\left(\widehat{I}_{t-1}\right)-g_{0}\left(I_{t-1}\right)\right|=o_{P}(1)$.

Now, from Theorem 1 we can construct ADF tests for $H_{0}$ as follows. Define $\sigma^{2}:=E\left[g_{e}^{2}\left(I_{t-1}, \theta_{0}\right)\right]$. The simbol $\Longrightarrow$ denotes weak convergence in the metric space $D([-\infty, \infty])$ of cadlag (right-continuous 
with left limits) functions on $[-\infty, \infty]$, endowed with the Skorohod metric, see Billingsley (1999). Notice that $R_{n, \widehat{g}}^{1}$ belongs to $D([-\infty, \infty])$, after defining $R_{n, \widehat{g}}^{1}(-\infty):=0$ and $R_{n, \widehat{g}}^{1}(+\infty):=0$.

Corollary 1: Under Assumptions A1, A3, A4, A6 and $H_{0}$,

$$
R_{n, \widehat{g}}^{1}(\cdot) \Longrightarrow \sigma B\left(F_{u}(\cdot)\right)
$$

where $B(\cdot)$ is a standard Brownian Bridge on $[0,1]$.

An application of the Continuous Mapping Theorem (CMT), see e.g. Theorem 1.3.6 in van der Vaart and Wellner (1996), and Lemma 3.1 in Chang (1990) yields

$$
C v M_{n}:=\int_{\mathbb{R}} \widehat{\sigma}^{-2}\left|R_{n, \widehat{g}}^{1}(x)\right|^{2} F_{n, u}(d x) \stackrel{d}{\longrightarrow} \int_{[0,1]}|B(x)|^{2} d x,
$$

and

$$
K S_{n}=\sup _{x \in \mathbb{R}}\left|\widehat{\sigma}^{-1} R_{n, \widehat{g}}^{1}(x)\right| \stackrel{d}{\longrightarrow} \sup _{u \in[0,1]}|B(u)|,
$$

where $\widehat{\sigma}^{2}=n^{-1} \sum_{t=1}^{n} \widehat{g}^{2}\left(\widehat{I}_{t-1}, \theta_{n}\right)$ estimates $\sigma^{2}$ and $F_{n, u}(\cdot)$ is the empirical cdf of the standardized residuals $\left\{\widehat{u}_{t}\right\}_{t=1}^{n}$.

The test statistics $C v M_{n}$ and $K S_{n}$ are very easy to compute, as $R_{n, \widehat{g}}^{1}$ takes at most $n$ different values which can be computed recursively by ordering the residuals. More concretely, denote by $\widehat{u}_{(1)} \leq \widehat{u}_{(2)} \leq \cdots \leq \widehat{u}_{(n)}$ the ordered residuals and by $\widehat{g}_{c(1)}, \ldots, \widehat{g}_{c(n)}$ the corresponding concomitants, i.e. $\widehat{g}_{c(j)}=\widehat{g}\left(\widehat{I}_{(j)-1}, \theta_{n}\right), 1 \leq j \leq n$. Then, it is straightforward to prove that

$$
C v M_{n}=\frac{1}{\widehat{\sigma}^{2} n^{2}} \sum_{t=1}^{n}\left(\sum_{j=1}^{t} \widehat{g}_{c(j)}\right)^{2}
$$

and

$$
K S_{n}=\max _{1 \leq t \leq n}\left|\frac{1}{\widehat{\sigma} \sqrt{n}} \sum_{j=1}^{t} \widehat{g}_{c(j)}\right| .
$$

Our new tests have well-known asymptotic distributions with critical values that are readily available, see e.g. Shorack and Wellner (1986). In particular, our Cramér-von Mises (CvM) and Kolmogorov (K) tests reject the null hypothesis at $5 \%$ if $C v M_{n}>0.46136$ and $K S_{n}>1.3581$, respectively.

\subsection{Power properties: choice of $g_{0}$}

In this section we discuss global and local power properties of tests based on $R_{n, \widehat{g}}^{1}$. Proofs of the main results in this section are omitted, as they follow from similar arguments to those of Theorem 1. They are available from the author upon request. Assume that under the alternative 
hypothesis there exists a pseudo-true value $\theta_{*} \in \Theta$ such that $\left|\theta_{n}-\theta_{*}\right|=o_{P}(1)$ and define $u_{t}\left(\theta_{*}\right)=$ $\left(Y_{t}-f\left(I_{t-1}, \theta_{*}\right)\right) / h\left(I_{t-1}, \theta_{*}\right)$

$$
d_{1 t *}:=\frac{h\left(I_{t-1}, \theta_{*}\right)-h\left(I_{t-1}\right)}{h\left(I_{t-1}\right)} \quad \text { and } \quad d_{2 t *}:=\frac{f\left(I_{t-1}, \theta_{*}\right)-f\left(I_{t-1}\right)}{h\left(I_{t-1}\right)} .
$$

Note that under the alternative $d_{1 t *} \neq 0$ and $d_{2 t *} \neq 0$ with positive probability. Similarly to the proof of Theorem 1, it can be shown that under the alternative, uniformly in $x \in \mathbb{R}$,

$$
n^{-1 / 2} R_{n, g}^{1}(x) \stackrel{P}{\longrightarrow} \Delta_{*}(x):=E\left[g_{e}\left(I_{t-1}, \theta_{*}\right) 1\left(u_{t}\left(\theta_{*}\right) \leq x\right)\right] .
$$

Thus, whether or not tests based on $R_{n, \widehat{g}}^{1}$ are consistent against fixed alternatives depends in turn on whether or not the function $\Delta_{*}(x)$ is different from the zero function. Note that $\Delta_{*}(x)=$ $E\left[g_{e}\left(I_{t-1}, \theta_{*}\right) F_{u}\left(x+x d_{1 t *}+d_{2 t *}\right)\right]$. It turns out that the function $\Delta_{*}(x)$ depends on the weight $g_{e}\left(I_{t-1}, \theta_{*}\right)$, on the probabilistic limit $\theta_{*}$, the specified and true models, through $d_{1 t *}$ and $d_{2 t *}$, and on the unknown cdf $F_{u}$. Hence, the function $\Delta_{*}(x)$ is in general unknown, what makes it difficult to study the global power properties of resulting tests in general cases.

The following example ${ }^{2}$ illustrates that the global consistency will depend in general on the choice of $g_{e}\left(I_{t-1}, \theta_{*}\right)$.

Example 1: Consider a Gaussian AR(1)-ARCH(1) model with mean and variance functions

$$
E\left[Y_{t} \mid I_{t-1}\right]=0.5 Y_{t-1}, \quad \operatorname{Var}\left[Y_{t} \mid I_{t-1}\right]=1+0.5 Y_{t-1}^{2},
$$

respectively, and Gaussian innovations $u_{t} \sim N(0,1)$. The econometrician specifies the model (1) with $f\left(I_{t-1}, \theta_{0}\right)=\theta_{01} Y_{t-1}$ and $h\left(I_{t-1}, \theta_{0}\right)=\theta_{02}, \theta_{0}=\left(\theta_{01}, \theta_{02}\right)^{\prime} \in \Theta \subset(-1,1) \times(0, \infty)$, and proceeds to estimate $\theta_{0}$ by the ordinary least squares $(O L S)$ estimator $\theta_{n}=\left(\theta_{n 1}, \theta_{n 2}\right)^{\prime}$. Under mild moment conditions, $\left|\theta_{n}-\theta_{*}\right|=o_{P}(1)$ with $\theta_{*}=(0.5, \sqrt{2})$. For this example,

$$
\Delta_{*}(x)=E\left[g_{e}\left(I_{t-1}, \theta_{*}\right) \Phi\left(x \frac{\sqrt{2}}{\sqrt{1+0.5 Y_{t-1}^{2}}}\right)\right],
$$

where, henceforth, $\Phi$ denotes the standard Gaussian cdf. Note that $X_{t}\left(\theta_{0}\right)=\left(1, Y_{t-1} / \theta_{02}\right)^{\prime}$. Therefore, if we choose $g_{0}\left(I_{t-1}\right)=Y_{t-1}^{3}$, hence $g_{e}\left(I_{t-1}, \theta_{*}\right)=Y_{t-1}^{3}-\gamma_{1} Y_{t-1}$, for a constant $\gamma_{1}$ that is not relevant for the following conclusions. Note that by symmetry,

$$
E\left[g_{e}\left(I_{t-1}, \theta_{*}\right) \mid Y_{t-1}^{2}\right]=0,
$$

which implies that $\Delta_{*}(x)$ will be identically zero, and the test will have no power. Other choices of $g_{0}$ for this example lead to consistent tests, such as $g_{0}\left(I_{t-1}\right)=Y_{t-1}^{2}$; see our Monte Carlo experiments below.

\footnotetext{
${ }^{2}$ We thank an anonymous referee for providing this example.
} 
Thus, this example shows that an unfortunate choice for $g_{0}$ may lead to inconsistency of the new tests. This problem is not specific to our tests and applies to other specification tests available in the literature. In particular, this drawback also appears in the tests by Wooldridge (1990), Koul and Ling (2006) and Stute et al. (2008), among others. This possible inconsistency is the price we pay for the ADF property and simplicity of our tests, and it can be mitigated by making the tests more complicated and losing the asymptotic pivotal property of the tests; see Section 5 for further discussion. Nevertheless, our Monte Carlo experiments below and other unreported simulations showed that for the alternatives considered and several choices of $g_{0}$ our tests have a satisfactory empirical power; see Section 4.

We now provide a detailed comparison with Wooldridge's (1990) test. It can be shown that Wooldridge's test based on (3) has global power provided

$$
\int y \Delta_{*}(d y) \neq 0 \quad \text { or } \quad \int y^{2} \Delta_{*}(d y) \neq 0 .
$$

Hence, we conclude that Wooldridge's (1990) test has no global power whenever our test has no global power (i.e. if $\left.\Delta_{*}(y) \equiv 0\right)$. The reciprocal is not true, as there are examples where our test has non-trivial power but Wooldridge's (1990) test has not. The following example illustrates this point; see also our application to the S\&P 500.

ExAmple 2: Consider a Gaussian AR(1) model with

$$
E\left[Y_{t} \mid I_{t-1}\right]=\frac{1}{\sqrt{2}} Y_{t-1}, \quad \operatorname{Var}\left[Y_{t} \mid I_{t-1}\right]=1
$$

respectively, and Gaussian innovations $u_{t} \sim N(0,1)$. The econometrician specifies the model (1) with $f\left(I_{t-1}, \theta_{0}\right)=\theta_{0}$ and $h\left(I_{t-1}, \theta_{0}\right)=1$, and proceeds to estimate $\theta_{0}$ by the sample mean $\theta_{n}$. Under mild moment conditions, $\left|\theta_{n}-\theta_{*}\right|=o_{P}(1)$ with $\theta_{*}=0$. For this example,

$$
\Delta_{*}(x)=E\left[g_{e}\left(I_{t-1}, \theta_{*}\right) \Phi\left(x-2^{-1 / 2} Y_{t-1}\right)\right] .
$$

Note that $X_{t}\left(\theta_{0}\right)=1$. Therefore, if we choose $g_{0}\left(I_{t-1}\right)=Y_{t-1}^{2}$, hence $g_{e}\left(I_{t-1}, \theta_{*}\right)=Y_{t-1}^{2}-1$. It is straightforward to show that, although $E\left[g_{e}\left(I_{t-1}, \theta_{*}\right) Y_{t-1}\right]=0$, the function $\Delta_{*}(x)$ is different from the zero function. Wooldridge's (1990) test for the mean will have no power against this alternative, whereas our tests is consistent.

Of course, we do not claim that our tests have always higher power than Wooldridge's (1990) tests; they are complementary rather than competing tests. Moreover, strictly speaking they cannot be compared since they are valid under different sets of assumptions. Wooldridge's (1990) tests are robust to deviations of the iid assumption in the innovations, whereas our tests are not. However, being based on a finite number of functions of residuals, see (3), Wooldridge's (1990) tests are not 
consistent against a large class of alternatives to $H_{0}$ that can be picked up by our tests, as they consider an infinite number of transformations of residuals indexed by $x \in \mathbb{R}$.

We now turn into the study of the local power of our tests. This study will shed some light into the problem of the choice of $g_{0}$. We shall show that the choice of $g_{0}$ allows us to construct ADF tests with good local power against desired alternatives, e.g. threshold alternatives. Consider the following local alternatives within the model (1):

$$
H_{1 n}: f(y)=f\left(y, \theta_{0}\right)+n^{-1 / 2} s_{m}(y) \text { and } h(y)=h\left(y, \theta_{0}\right),
$$

and

$$
H_{2 n}: f(y)=f\left(y, \theta_{0}\right) \text { and } h(y)=h\left(y, \theta_{0}\right)+n^{-1 / 2} s_{v}(y),
$$

Following the arguments in the proof of Theorem 1, one can show that under the local alternatives $H_{i n}, i=1,2$, the process $R_{n, \widehat{g}}^{1}$ converges to $\sigma B\left(F_{u}(\cdot)\right)+D_{i}(x)$, respectively, where for $i=1,2$,

$$
D_{1}(x)=E\left[g_{e}\left(I_{t-1}, \theta_{0}\right) s_{m}\left(I_{t-1}\right) h^{-1}\left(I_{t-1}, \theta_{0}\right)\right] f_{u}(x)
$$

and

$$
D_{2}(x)=E\left[g_{e}\left(I_{t-1}, \theta_{0}\right) s_{v}\left(I_{t-1}\right) h^{-1}\left(I_{t-1}, \theta_{0}\right)\right] x f_{u}(x) .
$$

It is then clear that the optimal choice for $g_{0}\left(I_{t-1}\right)$ under $H_{1 n}$ is the orthogonal projection of $s_{m}\left(I_{t-1}\right) h^{-1}\left(I_{t-1}, \theta_{0}\right)$ on the orthocomplement of the span generated by $X_{t}\left(\theta_{0}\right)$. A similar conclusion holds for $H_{2 n}$, details are omitted. Thus, we have shown that, provided $D_{i}(x) \neq 0$ for $i=1,2$, the tests have nontrivial local power.

\section{ARMA-GARCH MODELS}

This section verifies the applicability of our previous results for the well-known class of $\operatorname{ARMA}(P, Q)$ $\operatorname{GARCH}(p, q)$ models. An $\operatorname{ARMA}(P, Q)-\operatorname{GARCH}(p, q)$ process, $P, Q, p, q \in \mathbb{N}$, satisfies the equations

$$
\left\{\begin{array}{c}
Y_{t}=f\left(I_{t-1}, \vartheta_{0}\right)+\varepsilon_{t} \equiv c_{0}+\sum_{i=1}^{Q} a_{0 i} Y_{t-i}-\sum_{j=1}^{P} b_{0 j} \varepsilon_{t-j}+\varepsilon_{t}, \quad \varepsilon_{t}=h_{t} u_{t}, \\
h_{t}^{2} \equiv h^{2}\left(I_{t-1}, \theta_{0}\right)=w_{0}+\sum_{i=1}^{q} \alpha_{0 i} \varepsilon_{t-i}^{2}+\sum_{j=1}^{p} \beta_{0 j} h_{t-j}^{2}, \quad t \in \mathbb{Z},
\end{array}\right.
$$

where $u_{t}$ is a sequence of iid random variables independent of $I_{t-1}=\left(Y_{t-1}, Y_{t-2}, \ldots\right)^{\prime}$, and $w_{0}>$ $0, \alpha_{0 i} \geq 0, i=1, \ldots, q, \beta_{0 j} \geq 0, j=1, \ldots, p$. Denote a generic vector of parameters by $\vartheta=$ $\left(c, a_{1}, \ldots, a_{Q}, b_{1}, \ldots, b_{P}\right)^{\prime}, \varphi=\left(w, \alpha_{1}, \ldots, \alpha_{q}, \beta_{1}, \ldots, \beta_{p}\right)^{\prime}$ and $\theta=\left(\vartheta^{\prime}, \varphi^{\prime}\right)^{\prime}$, and the parameter space $\Theta \subset \mathbb{R}^{P+Q+1} \times(0,+\infty) \times[0,+\infty)^{p+q}$. The true parameter value is unknown and it is denoted by $\theta_{0}=\left(\vartheta_{0}^{\prime}, \varphi_{0}^{\prime}\right)^{\prime}$, with $\vartheta_{0}=\left(c_{0}, a_{01}, \ldots, a_{0 Q}, b_{01}, \ldots, b_{0 P}\right)^{\prime}$ and $\varphi_{0}=\left(w_{0}, \alpha_{01}, \ldots, \alpha_{0 q}, \beta_{01}, \ldots, \beta_{0 p}\right)^{\prime}$. We assume that $\theta_{0}$ belongs to the interior of $\Theta$ and that $\Theta$ is compact. 
For the stationarity of the model we define the matrix:

$$
A_{t}=\left[\begin{array}{cccccccc}
\alpha_{1} u_{t}^{2} & \cdots & \alpha_{q-1} u_{t}^{2} & \alpha_{q} u_{t}^{2} & \beta_{1} u_{t}^{2} & \cdots & \beta_{p-1} u_{t}^{2} & \beta_{p} u_{t}^{2} \\
1 & \cdots & 0 & 0 & 0 & \cdots & 0 & 0 \\
\vdots & \ddots & \vdots & \vdots & \vdots & \ddots & \vdots & \vdots \\
0 & \cdots & 1 & 0 & 0 & \cdots & 0 & 0 \\
\alpha_{1} & \cdots & \alpha_{q-1} & \alpha_{q} & \beta_{1} & \ldots & \beta_{p-1} & \beta_{p} \\
0 & \cdots & 0 & 0 & 1 & \cdots & 0 & 0 \\
\vdots & \ddots & \vdots & \vdots & \vdots & \ddots & \vdots & \vdots \\
0 & \cdots & 0 & 0 & 0 & \cdots & 1 & 0
\end{array}\right] .
$$

The spectral radius of a square matrix $A$ is denoted by $\rho(A)$. Denote by $\otimes$ the Kronecker product. Define the polynomials $\mathcal{A}_{\theta}(z)=\sum_{i=1}^{q} \alpha_{i} z^{i}, \mathcal{B}_{\theta}(z)=1-\sum_{j=1}^{p} \beta_{j} z^{i}, A_{\vartheta}(z)=\sum_{i=1}^{Q} a_{i} z^{i}, B_{\vartheta}(z)=$ $1-\sum_{j=1}^{P} b_{j} z^{i}, z \in \mathbb{C}$. By convention, $\mathcal{A}_{\theta}(z) \equiv 0$ if $Q=0$ and $\mathcal{B}_{\theta}(z) \equiv 1$ if $P=0$. Similarly for $A_{\vartheta}(z)$ and $B_{\vartheta}(z)$. The next assumption is sufficient for strictly stationarity of the model and for the asymptotic normality of the QMLE estimator (see Francq and Zakoïan (2004) and references therein).

Assumption B1: $u_{t}^{2}$ has a non-degenerate distribution. If $p>1(P>1), A_{\vartheta_{0}}(z)\left(\mathcal{A}_{\theta_{0}}(z)\right)$ and $B_{\vartheta_{0}}(z)$ $\left(\mathcal{B}_{\theta_{0}}(z)\right)$ have no common roots, $a_{0 P} \neq 0$ or $b_{0 Q} \neq 0, \mathcal{A}_{\theta_{0}}(1) \neq 0$ and $\alpha_{0 q}+\beta_{0 p} \neq 0$. Moreover, $\forall \theta \in \Theta, A_{\vartheta}(z) B_{\vartheta}(z)=0$ implies $|z|>1 . \rho\left(E\left[\left(A_{t} \otimes A_{t}\right)\right]\right)<1$ and $\forall \theta \in \Theta, \sum_{j=1}^{p} \beta_{j}<1$.

In particular, Chen and An (1998) showed that $\rho\left(E\left[\left(A_{t} \otimes A_{t}\right)\right]\right)<1$ is necessary and sufficient for the existence of a strictly stationarity and ergodicity solution to (10) with finite fourth moment. Assume we are given a sample of size $n$ of such stationary solution, $\left\{Y_{t}\right\}_{t=1}^{n}$ say. $\operatorname{ARMA}(P, Q)$ $\operatorname{GARCH}(p, q)$ models are usually estimated by the QMLE; see Li, Ling and McAleer (2002) and references therein. We describe the estimator as follows. If $q \geq Q$, the initial values $\widehat{I}_{0}=$ $\left(Y_{0}, \ldots, Y_{1-(q-Q)-P}, \widetilde{\varepsilon}_{-q+Q}, \ldots, \widetilde{\varepsilon}_{1-q}, \widetilde{\sigma}_{0}^{2}, \ldots, \widetilde{\sigma}_{1-p}^{2}\right)^{\prime}$ allow to compute $\widetilde{\varepsilon}_{t}(\vartheta)$, for $t=-q+Q+1, \ldots, n$, and $\widetilde{\sigma}_{t}^{2}(\theta)$, for $t=1, \ldots, n$, according to

$$
\left\{\begin{array}{c}
\widetilde{\varepsilon}_{t} \equiv \widetilde{\varepsilon}_{t}(\vartheta)=Y_{t}-c_{0}-\sum_{i=1}^{Q} a_{0 i} Y_{t-i}+\sum_{j=1}^{P} b_{0 j} \widetilde{\varepsilon}_{t-j}(\vartheta) \\
\widetilde{\sigma}_{t}^{2} \equiv \widetilde{\sigma}_{t}^{2}(\theta)=w+\sum_{i=1}^{q} \alpha_{i} \widetilde{\varepsilon}_{t-i}^{2}(\vartheta)+\sum_{j=1}^{p} \beta_{j} \widetilde{\sigma}_{t-j}^{2}(\theta)
\end{array}\right.
$$

When $q<Q$, the required initial values are $\widehat{I}_{0}=\left(Y_{0}, \ldots, Y_{1-(q-Q)-P}, \widetilde{\varepsilon}_{0}, \ldots, \widetilde{\varepsilon}_{1-Q}, \widetilde{\sigma}_{0}^{2}, \ldots, \widetilde{\sigma}_{1-p}^{2}\right)^{\prime}$. For simplicity, these initial values will be taken fixed (neither random nor function of parameters). Denote $\widehat{I}_{t}=\left(Y_{t}, \ldots, Y_{1}, \widehat{I}_{0}\right), t=1, \ldots n$.

A QMLE estimator is defined as any measurable solution $\widehat{\theta}_{n}$ of

$$
\widehat{\theta}_{n}=\underset{\theta \in \Theta}{\arg \min } \widetilde{L}_{n}(\theta)
$$


where

$$
\widetilde{L}_{n}(\theta)=n^{-1} \sum_{t=1}^{n} \widetilde{\ell}_{t}(\theta), \quad \widetilde{\ell}_{t}=\widetilde{\ell}_{t}(\theta)=\frac{\widetilde{\varepsilon}_{t}^{2}(\vartheta)}{\widetilde{\sigma}_{t}^{2}(\theta)}+\log \widetilde{\sigma}_{t}^{2}(\theta) .
$$

The asymptotic theory of $\widehat{\theta}_{n}$ has been studied by numerous authors. For instance, in the present context Francq and Zakoïan (2004) proved that under Assumption B1

$$
\sqrt{n}\left(\widehat{\theta}_{n}-\theta_{0}\right) \longrightarrow{ }_{d} N\left(0, J^{-1} I J^{-1}\right),
$$

where $J=E_{\theta_{0}}\left[\frac{\partial^{2} \ell_{t}\left(\theta_{0}\right)}{\partial \theta \partial \theta^{\prime}}\right], I=E_{\theta_{0}}\left[\frac{\partial \ell_{t}\left(\theta_{0}\right)}{\partial \theta} \frac{\partial \ell_{t}\left(\theta_{0}\right)}{\partial \theta^{\prime}}\right]$ (explicit expressions for $\ell_{t}(\theta), \partial \ell_{t}\left(\theta_{0}\right) / \partial \theta$ and $\partial^{2} \ell_{t}\left(\theta_{0}\right) / \partial \theta \partial \theta^{\prime}$ can be found in Francq and Zakoïan (2004)). Therefore, Assumption B1 is sufficient for Assumptions A1(a) and A3 here.

It can be shown (see displays (4.58-4.60) in Francq and Zakoïan (2004)) that under our Assumption B1

$$
E\left[\sup _{\theta \in \Theta_{0}}\left|\frac{\dot{f}_{t}(\theta)}{h\left(I_{t-1}, \theta_{0}\right)}\right|^{4}\right]<\infty, \quad E\left[\sup _{\theta \in \Theta_{0}}\left|\frac{\dot{h}_{t}(\theta)}{h\left(I_{t-1}, \theta_{0}\right)}\right|^{4}\right]<\infty,
$$

holds, so Assumption A2 (and A6(a)) is also satisfied. As for the initial conditions, $\rho\left(E\left[\left(A_{t} \otimes A_{t}\right)\right]\right)<$ 1 implies for a positive constant $\rho<1$,

$$
E \sup _{\theta \in \Theta_{0}}\left|\frac{h\left(\widehat{I}_{t-1}, \theta\right)-h\left(I_{t-1}, \theta\right)}{h\left(I_{t-1}\right)}\right|^{2}<C \rho^{t}, \quad E \sup _{\theta \in \Theta_{0}}\left|\frac{f\left(\widehat{I}_{t-1}, \theta\right)-f\left(I_{t-1}, \theta\right)}{h\left(I_{t-1}\right)}\right|^{2}<C \rho^{t} .
$$

Hence, Assumption A4 also holds. Therefore, we observe that our assumptions are naturally satisfied for ARMA-GARCH models.

Some remarks on how to compute our tests for ARMA-GARCH models are in order. First, we compute residuals according to

$$
\widehat{u}_{t}=\frac{\widetilde{\varepsilon}_{t}\left(\widehat{\vartheta}_{n}\right)}{\widetilde{\sigma}_{t}\left(\widehat{\theta}_{n}\right)}, \quad t=1, \ldots, n,
$$

using the QMLE $\widehat{\theta}_{n}=\left(\widehat{\vartheta}_{n}^{\prime}, \widehat{\varphi}_{n}^{\prime}\right)^{\prime}$ and the previous initial conditions. Expressions for the derivatives $\dot{f}_{t}\left(\theta_{0}\right)$ and $\dot{h}_{t}\left(\theta_{0}\right)$ can be found in e.g. Koul and Ling (2006), among others. We remark that for zero mean homokedastic ARMA models there is no need for running any preliminary regression. In this special case, any sample centered weight $g_{0}\left(I_{t-1}\right)$ will work since it will trivially satisfy $(4)$ and $(5)$.

\section{FINITE SAMPLE PERFORMANCE AND DATA ANALYSIS}

This section examines the performance through some Monte Carlo experiments of the test statistics $C v M_{n}$ and $K S_{n}$ for testing $H_{0}$. We compare the new tests with Wooldridge's (1990) test based on the quadratic form

$$
W_{n}=S_{n}^{\prime} \Sigma_{n}^{-1} S_{n}
$$


where $S_{n}=n^{-1 / 2} \sum_{t=1}^{n} \phi\left(I_{t}, \widehat{\theta}_{n}\right)$ and $\Sigma_{n}=n^{-1} \sum_{t=1}^{n} \phi\left(I_{t}, \widehat{\theta}_{n}\right) \phi^{\prime}\left(I_{t}, \widehat{\theta}_{n}\right)$, with $\phi\left(I_{t}, \widehat{\theta}_{n}\right)=\widehat{g}_{c}\left(I_{t-1}, \widehat{\theta}_{n}\right) \times$ $\left(\widehat{u}_{t}, \widehat{u}_{t}^{2}\right)^{\prime}$. Under the null hypothesis $H_{0}, W_{n}$ converges to a chi-squared distribution with two degrees of freedom. All the experiments are carried out using the GARCH Toolbox from Matlab 7.1.

First, we consider the data generating process and fitted model of Example 1 to illustrate how the choice of $g_{0}$ affects the power properties. Table 1 reports the rejection probabilities for several choices of the initial weight $g_{0}$ and sample sizes $n=100,500$ and 1000. As expected, our tests have little power against this alternative when asymmetric weights are chosen, even for large sample sizes as $n=1000$. However, for other choices of $g_{0}$ our tests present a satisfactory power performance, with moderate power for $g_{0}\left(I_{t-1}\right)=Y_{t-1} Y_{t-2}$ and $\varepsilon_{t-1}^{2} 1\left(\varepsilon_{t-1} \leq 0\right)$, and high power for $g_{0}\left(I_{t-1}\right)=Y_{t-1}^{2}$, $\cos \left(Y_{t-1}\right)$ and $\varepsilon_{t-1}^{2}$. Our tests outperform Wooldridge's (1990) test for almost all sample sizes and choices of $g_{0}$. This simulation also suggests that it may be important to try several choices of $g_{0}$ for power purposes.

\section{Please insert Table 1 about here}

In the second experiment, the following $\operatorname{AR}(1)-\operatorname{GARCH}(1,1)$ is used:

$$
Y_{t}=\mu+a Y_{t-1}+\varepsilon_{t}, \quad \varepsilon_{t}=h\left(I_{t-1}, \theta_{0}\right) u_{t}, \quad h^{2}\left(I_{t-1}, \theta_{0}\right)=\alpha_{00}+\alpha_{01} \varepsilon_{t-1}^{2}+\beta_{01} h^{2}\left(I_{t-1}, \theta_{0}\right),
$$

where $\left\{u_{t}\right\}$ are iid disturbances, the true parameters are $\theta_{0}=\left(\mu, a, \alpha_{00}, \alpha_{01}, \beta_{01}\right) \in \Theta$, with

$$
\Theta=\left\{\left(\mu, a, \alpha_{0}, \alpha_{1}, \beta_{1}\right) \in \mathbb{R}^{5}:|a| \leq \bar{a}<1, \alpha_{0} \geq c>0, \alpha_{1} \geq 0, \beta_{1} \geq 0 \text { and } \alpha_{2}+\alpha_{3} \leq \bar{\rho}<1\right\} .
$$

The null model in $H_{0}$ corresponds to $a=0$, that is, a pure GARCH $(1,1)$ model. We report in Figure 1 the rejection probabilities for model (12) with $a$ varying from -0.9 to 0.9 in increments of 0.1 , and $\left(\mu, \alpha_{0}, \alpha_{1}, \beta_{1}\right)=(0,0.025,0.25,0.5)$. The empirical power functions are plotted in Figure 1 for $n=100$ and the innovations' distributions $N(0,1)$ and $t$-student with 3 degrees of freedom. We consider 1000 replications in all experiments. For this experiment the initial weight function $g_{0}$ is taken to be $g_{0}\left(I_{t-1}\right)=Y_{t-1}, t \in \mathbb{Z}$. Other choices of $g_{0}$ were considered but not reported for the sake of space. They are available from the author upon request.

\section{Please insert Figure 1 about here}

From Figure 1 we see that the size performance is satisfactory. The rejection probabilities for the Gaussian distribution for $C v M_{n}, K S_{n}$ and $W_{n}$ are, respectively, 0.057, 0.037 and 0.050. For the $t$ distribution with 3 degrees of freedom, the rejection probabilities are, respectively, 0.065, 0.041, and 0.035. These simulations show the robustness of our tests to fat-tail distributions. The empirical power properties of our tests are excellent against these alternatives already for as small samples sizes as $n=100$. For Gaussian innovations, $C v M_{n}$ outperforms $W_{n}$, which in turn has higher power 
than $K S_{n}$. For $t$-student innovations our tests have higher power than Wooldridge's (1990) test uniformly in all values of $c$, with $C v M_{n}$ attaining the highest rejection probabilities.

In the third experiment we consider some nonlinear alternatives to the $\operatorname{AR}(1)-\operatorname{GARCH}(1,1)$ model (12) with mean parameters $(\mu, a)=(0.02,0.02)$ and variance parameters $\left(\alpha_{0}, \alpha_{1}, \beta_{1}\right)=$ $(0.08,0.1,0.85)$. These parameter values are motivated from our application. We denote the null model by A0. The alternatives considered are:

ALT1: $\operatorname{ARMA}(1,1)-\operatorname{GARCH}(1,1)$ model: $Y_{t}=0.02+0.02 Y_{t-1}+0.5 \varepsilon_{t-1}+\varepsilon_{t}$.

ALT2: TAR model: $Y_{t}=0.6 Y_{t-1}+\varepsilon_{t}$ if $Y_{t-1} \leq 1$ and $Y_{t}=-0.5 Y_{t-1}+\varepsilon_{t}$ if $Y_{t-1}>1$.

ALT3: $\operatorname{EGARCH}(1,1)$ model: $Y_{t}=h_{t} u_{t}, \ln h_{t}^{2}=0.025+0.5 \ln h_{t-1}^{2}+0.25\left(\left|u_{t-1}\right|-(2 / \pi)^{1 / 2}\right)-0.8 u_{t-1}$.

ALT4: Bilinear model (BIL): $Y_{t}=0.6 Y_{t-1}+0.7 u_{t-1} Y_{t-2}+u_{t}$.

ALT5: Non-Linear Moving Average model (NLMA): $Y_{t}=0.8 u_{t-1}^{2}+u_{t}$.

In models ALT1-ALT5, $\varepsilon_{t}$ is as in $(12)$ with $\left(\alpha_{0}, \alpha_{1}, \beta_{1}\right)=(0.08,0.1,0.85)$ and $\left\{u_{t}\right\}$ iid $N(0,1)$ variates. Table 2 shows the rejection probabilities for these alternatives for $n=300, n=600$ and $n=1000$, when $g_{0}\left(I_{t-1}\right)=Y_{t-1}$.

\section{Please insert Table 2 about here}

Again, Table 2 reveals that the size performance of our tests is satisfactory. $K S_{n}$ presents some slight underrejection. It is shown that our tests are able to detect all the linear and nonlinear alternatives considered. The highest power is attained at ALT5. Again, $C v M_{n}$ presents the highest empirical power. $K S_{n}$ has higher power than $W_{n}$ against ALT3, ALT4 and ALT5, whereas in general the reciprocal is true for ALT1 and ALT2. Unreported simulations confirm that the excellent empirical power and size performance observed extend to other parameter values $\left(\alpha_{0}, \alpha_{1}, \beta_{1}\right)$ in the stationary region. These simulations indicate that the proposed tests have satisfactory size and power performance in moderate finite-samples and compare favorably with competing tests, thereby providing useful model checks for ARMA-GARCH models.

We next apply our testing methodology to the well-known and extensively studied S\&P500 daily stock index. The debate on whether the dynamics of economic and financial time series are determined by the conditional mean or the conditional variance has important implications on many applications including portfolio selection and asset pricing. The S\&P500 daily stock index is a representative of the data for which the GARCH model has been extensively used, see e.g. Bollerslev, et al. (1992), Bera and Higgins (1997) and references therein. We consider several periods of this stock index. The first period spans from January 21990 until December 31 1993, the second period 
from January 31994 until December 311997 and the third from January 21998 until August 28 2002. The martingale properties of these samples were studied in Escanciano and Velasco (2006). The number of observations in each period is 1013, 1011 and 1170, respectively. A common feature of financial data is that the performance of any model is not consistent across different sub-periods of the sample, suggesting that the correct model is period-specific, see e.g. Loudon, Watt and Yadav (2000).

In this application we fit an $\mathrm{AR}(1)-\mathrm{GARCH}(1,1)$ model to the log differences of the $\mathrm{S} \& \mathrm{P} 500\left(Y_{t}\right)$ as in (12). We estimate the parameters with the QMLE and the results are reported in Table 3, and, as usual, the standard errors are in parenthesis.

\section{Please insert Table 3 about here}

From Table 3 we observe that the mean equation parameters are statistically insignificant in the first and third periods. The latter fact is consistent with the results obtained by Escanciano and Velasco (2006), who found evidence that in the periods 1990-1993 and 1998-2002 the S\&P500 return series is a martingale difference sequence. In the same study, these authors provided evidence that for the period 1994-1997 the S\&P500 is not a martingale difference sequence, which is again consistent with the results of Table 3. Comparing the different periods, we observe a substantial decrease in the persistence of the series.

We proceed by fitting an $\mathrm{AR}(1)-\mathrm{GARCH}(1,1)$ model as in (12) for the period 1994-1997 and a pure GARCH(1,1) model for the periods 1990-1993 and 1998-2002. We apply our tests and Wooldridge's (1990) test to this data sets with several initial weights $g_{0}\left(I_{t-1}\right)$. We report the values of the test statistics $C v M_{n}, K S_{n}$ and $W_{n}$ in Table 4.

Please insert Table 4 about here

We summarize our findings as follows: We reject the null hypothesis of $\operatorname{GARCH}(1,1)$ specification with both statistics $C v M_{n}$ and $K S_{n}$ at the $5 \%$ level for essentially all cases within the first period. Wooldridge's (1990) test fails to detect this alternative. Thus, it is shown that a GARCH(1,1) specification for the conditional variance is not adequate for the first period. We conclude that weights involving the first lagged value of the dependent variable or the first lagged conditional mean error are sufficient to account for the lack-of-fit of the GARCH model. Further misspecifications at higher order lags can be investigated similarly. The $\operatorname{AR}(1)-\operatorname{GARCH}(1,1)$ and $\operatorname{GARCH}(1,1)$ specifications for the periods 1994-1997 and 1998-2002 seem satisfactory and no significative test statistic is found at the $5 \%$ level with the weights considered. Only the CvM test at $10 \%$ uncovers some discrepancy with the assumed model in the last period for the asymmetric weight. Unreported simulations confirm our findings with other weights. In particular we have also considered standardized weights 
$g_{0}\left(I_{t-1}\right) h^{-1}\left(I_{t-1}, \theta_{0}\right)$, where $g_{0}\left(I_{t-1}\right)$ is as in Table 4 . The results with these standardized weights are similar to those with the unstandardized ones, and hence they are omitted.

\section{CONCLUSIONS AND FUTURE WORK}

In this paper we have proposed ADF diagnostic tests for the adequacy of a class of heteroskedastic time series models, including but not restricted to ARMA-GARCH models. We have shown that our tests perform well in finite samples, comparing favorably with more traditional methods such as the tests proposed by Wooldridge (1990). Unlike most tests available in the literature that use empirical processes of covariates weighted by residuals, our tests are based on empirical processes of residuals weighted by appropriate transformations of the possible infinite-dimensional conditioning set. This fact allows us to construct tests that are not affected by the so-called curse of dimensionality in regression model checks. This property is essential if the interest is in non-Markovian models such as ARMA-GARCH models, where the conditioning set can be infinite-dimensional.

The weights in the weighted residual process are orthogonal to the score functions of the model. We show that this orthogonality renders to tests that are ADF. In addition to the ADF property and robustness to high-dimensional conditioning set, other appealing property of our tests is their computational simplicity (running an OLS estimation and ordering the resulting residuals is what is needed to compute the test statistics). The "price" we pay for these appealing properties is that an unfortunate choice of $g_{0}$, as well as the particular features of the unknown data generating process and the specified model, may lead to inconsistency of our tests; see Example 1. In fact, as shown recently by Escanciano (2009) this price is, in practice (i.e. in finite samples), paid by all omnibus specification tests for regression models, including the consistent ones. It is important to stress that this weakness can be also seen as its strength, since unlike with consistent tests, with our tests the local power properties are "controlled" by the researcher through the choice of $g_{0}$; whereas, unlike fully directional tests (e.g. Lagrange Multiplier tests), our tests may have global power against a large class of alternatives; see Example 2. Thus, our tests provide an interesting compromise between consistent and directional tests.

Two extensions of our methods can be shown to be useful in improving the power properties of our tests, although at the expense of losing the ADF property. First, a simple and trivial extension is to multivariate weights $g_{0}$. The principal components decomposition in Stute et al. (2008) can be considered as a method to combine (asymptotic independent) information from different weight functions into a single statistic. The second, and more complicated extension is to weights that are function-valued. For instance, if we take $g_{0}\left(I_{t-1}\right)=1\left(Y_{t-1} \leq y\right)$ as a function indexed by $y \in \mathbb{R}$, 
then our theory would lead to tests with possibly better power properties, based on the process

$$
R_{n, \widehat{g}}^{1}(x, y)=\frac{1}{\sqrt{n}} \sum_{t=1}^{n}\left\{1\left(Y_{t-1} \leq y\right)-\widehat{\gamma}_{n}^{\prime}\left(\theta_{n}, y\right) \widehat{X}_{t}\left(\theta_{n}\right)\right\} 1\left(\widehat{u}_{t} \leq x\right),
$$

with $\widehat{\gamma}_{n}^{\prime}\left(\theta_{n}, y\right)$ denoting the OLS estimator in the regression of $1\left(Y_{t-1} \leq y\right)$ against $\widehat{X}_{t}\left(\theta_{n}\right)$. Similarly to Theorem 1, it can be proved that under the conditions of Corollary 1 ,

$$
R_{n, \widehat{g}}^{1}(x, y)=\frac{1}{\sqrt{n}} \sum_{t=1}^{n} g_{e}\left(I_{t-1}, \theta_{0}, y\right)\left[1\left(u_{t} \leq x\right)-F_{u}(x)\right]+o_{P}(1),
$$

where $g_{e}\left(I_{t-1}, \theta_{0}, y\right)=1\left(Y_{t-1} \leq y\right)-\gamma_{0}\left(\theta_{0}, y\right) X_{t}\left(\theta_{0}\right)$. Unlike $R_{n, \widehat{g}}^{1}(x)$, the null limit of $R_{n, \widehat{g}}^{1}(x, y)$ is a Gaussian process with zero mean and complicated covariance function. The asymptotic null distribution of functionals of $R_{n, \widehat{g}}^{1}(x, y)$ can be approximated by bootstrap methods such as the multiplier-type bootstrap described in van der Vaart and Wellner (1996). The power properties of the resulting tests will be investigated elsewhere, in comparison with the simpler and still powerful tests proposed in the present paper. 


\section{PROOFS}

\subsection{Some general results}

In order to handle initial conditions we need to extend some general results in Koul and Ling (2006). More concretely, assumption (4.10) in Lemma 4.1 in Koul and Ling (2006) seems to be a strong assumption when initial conditions are considered; see the discussion before Theorem 1. To that end, we shall introduce a weak convergence theorem which generalizes Theorem 2.2.5 in Koul (2002) in several aspects. Most notably, unlike the aforementioned results, our weak convergence theorem allows us to consider initial conditions and to study the behaviour of our tests under the alternative hypothesis.

We need some notation. For each $n \geq 1,\left\{u_{n, t}\right\}_{t=1}^{n}$ are iid variables with cdf $F_{u}$. Let $\mathcal{F}_{n, t-1}$ the $\sigma$ field generated by the random variables $\left\{Y_{n, s}\right\}_{s=1}^{t-1}$ and $\left\{u_{n, s}\right\}_{s=1}^{t-1}, \mathcal{F}_{n, t-1}:=\sigma\left(Y_{n, t-1}, u_{n, t-1}, \ldots, Y_{n, 1}, u_{n, 1}\right)$. Denote by $\left(\Omega_{n}, \mathcal{A}_{n}, P_{n}\right), n \geq 1$, the probability space in which all the r.v.'s $\left\{u_{n, t}, Y_{n, t}\right\}_{t=1}^{n}$ are defined. Let $\|\cdot\|_{2, n}$ indicate the $L_{2}\left(P_{n}\right)$ norm. Assume that $u_{n, t}$ is independent of $\mathcal{F}_{n, t-1}$ for all $1 \leq t \leq n$ and all $n \in \mathbb{N}$. The reader is referred to Definitions 2.1.5 and 2.1.6 in van der Vaart and Wellner (1996) for definitions of the bracketing number $N_{[]}\left(\varepsilon, \mathcal{F},\|\cdot\|_{2}\right)$ and the covering number $N(\varepsilon, \mathcal{F},\|\cdot\|)$ with respect to a pseudo-norm $\|\cdot\|$.

Define the sets $\overline{\mathbb{R}}=[-\infty, \infty]$ and $\Delta_{K}=\left\{v \in \mathbb{R}^{p}:|v| \leq K\right\}$ for an arbitrary but fixed constant $K>0$. For positive finite constants $b>0$ and $c>0$ define the set $\mathcal{Z}_{b, c}=\left\{\left(z_{1}, z_{2}\right)^{\prime} \in \mathbb{R}^{2}:\left|z_{1}\right|+\left|z_{2}\right| \leq\right.$ $\left.b, z_{1}>-1+c\right\}$. Let $\left\{d_{1 t n}(v), d_{2 t n}(v), g_{n, t}(v)\right\}_{t=1}^{n}$ be a sequence of r.v's such that $d_{1 t n}(v), d_{2 t n}(v)$ and $g_{n, t}(v)$ are $\mathcal{F}_{n, t-1}-$ measurable for each $v \in \mathbb{R}^{p}$. For a given $K>0$ and with $z=\left(z_{1}, z_{2}\right)^{\prime} \in \mathbb{R}^{2}$ define

$$
\rho_{K}\left(x_{1}, x_{2}\right):=\sup _{|z| \leq K}\left|F_{u}\left(x_{1}+x_{1} z_{1}+z_{2}\right)-F_{u}\left(x_{2}+x_{2} z_{1}+z_{2}\right)\right|, \quad x_{1}, x_{2} \in \overline{\mathbb{R}} .
$$

Henceforth, to simplify notation we write $(x, v)$ instead of $\left(x, v^{\prime}\right)^{\prime}$. Let $d_{K}\left(u_{1}, u_{2}\right):=\rho_{K}\left(x_{1}, x_{2}\right)+$ $\left|v_{1}-v_{2}\right|$, where $u_{1}=\left(x_{1}, v_{1}\right)$ and $u_{2}=\left(x_{2}, v_{2}\right)$ belong to $\Pi_{K}:=\overline{\mathbb{R}} \times \Delta_{K}$.

For $(x, v) \in \Pi_{K}$ define $i_{n t}(x, v):=x+x d_{1 t n}(v)+d_{2 t n}(v)$ and the process

$$
V_{n}(x, v):=\frac{1}{n} \sum_{t=1}^{n} g_{n, t}(v)\left\{1\left(u_{n, t} \leq i_{n t}(x, v)\right)-F_{u}\left(i_{n t}(x, v)\right)\right\},
$$

indexed by $\gamma=(x, v) \in \Pi_{K}$. Consider the following assumptions:

W1 For any $0<\delta<1$,

$$
\max _{1 \leq t \leq n, n \geq 1} E\left[\sup _{\left|v_{1}-v_{2}\right| \leq \delta}\left|g_{n, t}\left(v_{1}\right)-g_{n, t}\left(v_{2}\right)\right|\right]<C \delta
$$

and

$$
\sup _{\varepsilon \in(0,1) \cap \mathbb{Q}^{1 \leq t \leq n}} \max n^{-1} \sum_{t=1}^{n} \varepsilon^{-2} \sup _{\left|v_{1}-v_{2}\right| \leq \varepsilon^{2}}\left|g_{n, t}\left(v_{1}\right)-g_{n, t}\left(v_{2}\right)\right|^{2}=O_{P}(1) \text {. }
$$


Moreover, for each $K>0, \max _{1 \leq t \leq n, n \geq 1} E\left[\bar{g}_{n, t, K}^{2}\right]<\infty$ and $n^{-1} \sum_{t=1}^{n} \bar{g}_{n, t, K}^{2}=O_{P_{n}}(1)$, where $\bar{g}_{n, t, K}=\sup _{v \in \Delta_{K}}\left|g_{n, t}(v)\right|$.

W2 $\left\{d_{1 t n}(\cdot), d_{2 t n}(\cdot): 1 \leq t \leq n\right\}$ are such that for each $K>0$ and all $\delta>0$

$$
\begin{gathered}
\sup _{v \in \Delta_{K}} \max _{1 \leq t \leq n}\left|d_{1 t n}(v)\right|+\left|d_{2 t n}(v)\right|=O_{P_{n}}(1), \\
\operatorname{Pr}\left(\inf _{v \in \Delta_{K}} \min _{1 \leq t \leq n} d_{1 t n}(v)>c-1\right) \geq 1-\delta, \quad \text { for some } c>0, \\
\max _{1 \leq t \leq n, n \geq 1} E\left[\sup _{\left|v_{1}-v_{2}\right| \leq \delta}\left|d_{i t n}\left(v_{1}\right)-d_{i t n}\left(v_{2}\right)\right|\right]<C \delta, \quad i=1,2,
\end{gathered}
$$

and

$$
\sup _{\varepsilon \in(0,1) \cap \mathbb{Q}} n^{-1} \sum_{t=1}^{n} \varepsilon^{-1} \bar{g}_{n, t, K}^{2}\left[\sup _{\left|v_{1}-v_{2}\right| \leq \varepsilon}\left|d_{i t n}\left(v_{1}\right)-d_{i t n}\left(v_{2}\right)\right|\right]=O_{P_{n}}(1), \quad i=1,2 .
$$

W3 $F_{u}$ has an absolutely continuous density $f_{u}$ such that for all $0<b<\infty$ and $0<c<\infty$,

$$
\sup _{x \in \mathbb{R},\left(z_{1}, z_{2}\right)^{\prime} \in \mathcal{Z}_{b, c}}|x| f_{u}\left(x+x z_{1}+z_{2}\right)<\infty .
$$

Theorem A1: Under the assumptions $\mathrm{W} 1-\mathrm{W} 3$, the process $V_{n}(\gamma)$ is asymptotically tight with respect to $\gamma=(x, v) \in \Pi_{K}$.

Proof of Theorem A1: We shall apply Theorem A1 in Delgado and Escanciano (2007) with (following their notation)

$$
w_{n t}(\gamma)=g_{n, t}(v)\left\{1\left(u_{n, t} \leq i_{n t}(x, v)\right)-F_{u}\left(i_{n t}(x, v)\right)\right\}, \quad \gamma=(x, v) \in \Pi_{K} .
$$

Define the class of functions $\mathcal{K}_{n}=\left\{w_{n} \cdot(\gamma): \gamma=(x, v) \in \Pi_{K}\right\}$ and fix $\gamma_{1}=\left(x_{1}, v_{1}\right) \in \Pi_{K}$. By W2, for any $\delta>0$, there exists $K>0$ and $c>0$ such that

$$
P\left(A_{n, K}\right) \geq 1-\delta, \quad P\left(B_{n, c}\right) \geq 1-\delta
$$

where $A_{n, K} \equiv\left\{\sup _{v \in \Delta_{K}} \max _{1 \leq t \leq n}\left|d_{1 t n}(v)\right|+\left|d_{2 t n}(v)\right|<K\right\}$ and $B_{n, c} \equiv\left\{\inf _{v \in \Delta_{K}} \inf _{1 \leq t \leq n} d_{1 t n}(v)>c-1\right\}$. 
Now, by Cauchy-Schwartz inequality and triangle inequality, for any $0<\delta<1$,

$$
\begin{aligned}
& \sup _{n \geq 1} E\left[\sup _{\substack{\gamma_{2} \in \Pi_{K} \\
d_{K}\left(u_{1}, u_{2}\right) \leq \delta}}\left|w_{n t}\left(\gamma_{1}\right)-w_{n t}\left(\gamma_{2}\right)\right|\right] \\
\leq & \left.C\left(\sup _{n \geq 1} E\left|g_{n, t}\left(v_{1}\right)\right|^{2}\right)^{1 / 2}\left(\sup _{n \geq 1} E\left[\sup _{u_{2}: d_{K}\left(u_{1}, u_{2}\right) \leq \delta} \mid F_{u}\left(i_{n t}\left(x_{1}, v_{1}\right)\right)\right)-F_{u}\left(i_{n t}\left(x_{2}, v_{2}\right)\right) \mid\right]\right)^{1 / 2} \\
& +C \sup _{n \geq 1}\left[\sup _{\left|v_{1}-v_{2}\right| \leq \delta}\left|g_{n, t}\left(v_{1}\right)-g_{n, t}\left(v_{2}\right)\right|\right] \\
\leq & \left.C\left(\sup _{n \geq 1}\left[\sup _{x_{2}: \rho_{K}\left(x_{1}, x_{2}\right) \leq \delta} \mid F_{u}\left(i_{n t}\left(x_{1}, v_{1}\right)\right)\right)-F_{u}\left(i_{n t}\left(x_{2}, v_{1}\right)\right) \mid 1\left(A_{n, K}\right)\right]\right)^{1 / 2}+ \\
& \left.+\left(\sup _{n \geq 1}\left[\sup _{u_{2}: d_{K}\left(u_{1}, u_{2}\right) \leq \delta} \mid F_{u}\left(i_{n t}\left(x_{2}, v_{1}\right)\right)\right)-F_{u}\left(i_{n t}\left(x_{2}, v_{2}\right)\right) \mid 1\left(B_{n, c}\right)\right]\right)^{1 / 2}+C \delta^{1 / 2} \\
\leq & C \delta^{1 / 2},
\end{aligned}
$$

where the last inequality is due to $\mathrm{W} 2, \mathrm{~W} 3$ and the definition of $\rho_{K}$.

The proof of Theorem 3 in Chen, Linton and van Keilegom (2003) yields that

$$
\sup _{n \geq 1} N_{[\cdot]}\left(\varepsilon, \mathcal{K}_{n},\|\cdot\|_{2, n}\right) \leq N\left((\varepsilon / 2 C)^{2}, \overline{\mathbb{R}}, \rho_{K}\right) \times N\left((\varepsilon / 2 C)^{2}, \Delta_{K},|\cdot|\right) .
$$

Hence, from equation (4.7) in Koul and Ling (2006) and the last display it follows that

$$
\sup _{n \geq 1} \int_{0}^{\infty}\left(\log \left(N_{[\cdot]}\left(\varepsilon, \mathcal{K}_{n},\|\cdot\|_{2, n}\right)\right)\right)^{1 / 2} d \varepsilon<\infty .
$$

Let $\mathcal{B}_{\varepsilon}=\left\{B_{k} ; 1 \leq k \leq N_{\varepsilon} \equiv \sup _{n \geq 1} N_{[]}\left(\varepsilon, \mathcal{K}_{n},\|\cdot\|_{2, n}\right\}\right.$, with $B_{k}=\left[\underline{w}_{k, n t}(\cdot), \bar{w}_{k, n t}(\cdot)\right]$, be the partition of $\mathcal{K}_{n}$ in $\varepsilon$-brackets with respect to $\|\cdot\|_{2, n}$, which is bounded by the corresponding product of $(\varepsilon / 2 C)^{2}-$ nets of $\Delta_{K}$ and $\overline{\mathbb{R}}$ implicit in the proof of Theorem 3 in Chen et al. (2003). Arguing as in (17), the conditional quadratic variation of the empirical process $V_{n}$ on the previous partition is

$$
\begin{aligned}
V_{n}\left(\mathcal{B}_{\varepsilon}\right)= & \max _{1 \leq k \leq N} n^{-1} \sum_{t=1}^{n} E\left[\sup _{\gamma_{1}, \gamma_{2} \in B_{k}}\left|w_{n t}\left(\gamma_{1}\right)-w_{n t}\left(\gamma_{2}\right)\right|^{2} \mid \mathcal{F}_{n, t-1}\right] \\
\leq & \left.C n^{-1} \sum_{t=1}^{n} \bar{g}_{n, t, K}^{2}\left[\sup _{d_{K}\left(u_{1}, u_{2}\right) \leq(\varepsilon / 2 C)^{2}} \mid F_{u}\left(i_{n t}\left(x_{1}, v_{1}\right)\right)\right)-F_{u}\left(i_{n t}\left(x_{2}, v_{2}\right)\right) \mid\right] \\
& +C n^{-1} \sum_{t=1}^{n} \sup _{\left|v_{1}-v_{2}\right| \leq(\varepsilon / 2 C)^{2}}\left|g_{n, t}\left(v_{1}\right)-g_{n, t}\left(v_{2}\right)\right|^{2} \\
\leq & O_{P}\left(\varepsilon^{2}\right)+C n^{-1} \sum_{t=1}^{n} \bar{g}_{n, t, K}^{2}\left[\sup _{\left|v_{1}-v_{2}\right| \leq(\varepsilon / 2 C)^{2}}\left|d_{1 t n}\left(v_{1}\right)-d_{1 t n}\left(v_{2}\right)\right|\right] \\
& +C n^{-1} \sum_{t=1}^{n} \bar{g}_{n, t, K}^{2}\left[\sup _{\left|v_{1}-v_{2}\right| \leq(\varepsilon / 2 C)^{2}}\left|d_{2 t n}\left(v_{1}\right)-d_{2 t n}\left(v_{2}\right)\right|\right]
\end{aligned}
$$


Therefore, the last display and W2 imply (14) in Delgado and Escanciano (2007, p. 669) for the partition $\mathcal{B}_{\varepsilon}$. Therefore, Theorem A1 in Delgado and Escanciano (2007) applies and the asymptotic tightness of $V_{n}(\gamma)$ follows.

\subsection{Proofs of the main results}

Proof of Theorem 1. Define

$$
d_{1 t n}(v):=\frac{h\left(\widehat{I}_{t-1}, \theta_{0}+n^{-1 / 2} v\right)-h\left(I_{t-1}, \theta_{0}\right)}{h\left(I_{t-1}, \theta_{0}\right)}, \quad d_{2 t n}(v):=\frac{f\left(\widehat{I}_{t-1}, \theta_{0}+n^{-1 / 2} v\right)-f\left(I_{t-1}, \theta_{0}\right)}{h\left(I_{t-1}, \theta_{0}\right)} .
$$

To simplify notation we write $i_{n, t}(x, v)=x+x d_{1 t n}(v)+d_{2 t n}(v)$.

Define the process

$$
K_{n}(x, v)=\frac{1}{\sqrt{n}} \sum_{t=1}^{n} \widehat{g}_{n, t}(v)\left\{1\left(u_{t} \leq i_{n, t}(x, v)\right)-F_{u}(x)\right\}
$$

with $\widehat{g}_{n, t}(v)=g\left(\widehat{I}_{t-1}, \theta_{0}+n^{-1 / 2} v\right)-E\left[g\left(I_{t-1}, \theta_{0}\right)\right]$. By A4, uniformly in $\gamma=(x, v) \in \Pi_{K}$,

$$
K_{n}(x, v)=\frac{1}{\sqrt{n}} \sum_{t=1}^{n} g_{n, t}(v)\left\{1\left(u_{t} \leq i_{n, t}(x, v)\right)-F_{u}(x)\right\}+o_{P}(1),
$$

where $g_{n, t}(v)=g\left(I_{t-1}, \theta_{0}+n^{-1 / 2} v\right)-E\left[g\left(I_{t-1}, \theta_{0}\right)\right]$. Now, write

$$
\begin{aligned}
K_{n}(x, v)= & \frac{1}{\sqrt{n}} \sum_{t=1}^{n} g_{n, t}(v)\left[1\left(u_{t} \leq x\right)-F_{u}(x)\right] \\
& +\frac{1}{\sqrt{n}} \sum_{t=1}^{n} g_{n, t}(v)\left[F_{u}\left(i_{n, t}(x, v)\right)-F_{u}(x)\right] \\
& +\frac{1}{\sqrt{n}} \sum_{t=1}^{n} g_{n, t}(v)\left[1\left(u_{t} \leq i_{n, t}(x, v)\right)-1\left(u_{t} \leq x\right)-F_{u}\left(i_{n, t}(x, v)\right)+F_{u}(x)\right]+o_{P}(1) \\
: \quad= & A_{1 n}(x, v)+A_{2 n}(x, v)+A_{3 n}(x, v)+o_{P}(1) .
\end{aligned}
$$

To study the asymptotic behaviour of $A_{3 n}(x, v)$ we shall apply Theorem A1 to the process:

$$
\widetilde{K}_{n}(x, v):=\frac{1}{\sqrt{n}} \sum_{t=1}^{n} g_{n, t}(v)\left[1\left(u_{t} \leq i_{n, t}(x, v)\right)-F_{u}\left(i_{n, t}(x, v)\right)\right] .
$$

We proceed to verify W1-W3 for this particular example. Assumption W3 is directly assumed in A1(b). Assumption A5 and the Ergodic Theorem implies W1. It remains to verify W2.

For a sufficiently large $n$, A4 implies, uniformly in $v \in \Delta_{K}$,

$$
\begin{aligned}
\max _{1 \leq t \leq n}\left|d_{1 t n}(v)\right| \leq & \max _{1 \leq t \leq n}\left|\frac{h\left(\widehat{I}_{t-1}, \theta_{0}+n^{-1 / 2} v\right)-h\left(I_{t-1}, \theta_{0}+n^{-1 / 2} v\right)}{h\left(I_{t-1}, \theta_{0}\right)}\right| \\
& +\max _{1 \leq t \leq n}\left|\frac{h\left(I_{t-1}, \theta_{0}+n^{-1 / 2} v\right)-h\left(I_{t-1}, \theta_{0}\right)}{h\left(I_{t-1}, \theta_{0}\right)}\right| \\
\leq & O_{P}(1)+K n^{-1 / 2} \max _{1 \leq t \leq n} \sup _{\theta \in \Theta_{0}}\left|\frac{\dot{h}_{t}(\theta)}{h\left(I_{t-1}, \theta_{0}\right)}\right|=O_{P}(1) .
\end{aligned}
$$


Similarly, it can be proved that $\sup _{v \in \Delta_{K}} \max _{1 \leq t \leq n}\left|d_{2 t n}(v)\right|=O_{P}(1)$. This proves (13).

Note that

$$
\inf _{v \in \Delta_{K}} \min _{1 \leq t \leq n} \frac{h\left(\widehat{I}_{t-1}, \theta_{0}+n^{-1 / 2} v\right)}{h\left(I_{t-1}, \theta_{0}\right)}-1>c-1
$$

provided

$$
\max _{1 \leq t \leq n} \sup _{\theta \in \Theta_{0}}\left|\frac{h\left(I_{t-1}, \theta_{0}\right)}{h\left(\widehat{I}_{t-1}, \theta\right)}\right|<c^{-1},
$$

which holds with high probability by choosing a suitable $c>0$, because $\max _{1 \leq t \leq n} \sup _{\theta \in \Theta_{0}}\left|h\left(I_{t-1}, \theta_{0}\right) / h\left(\widehat{I}_{t-1}, \theta\right)\right|=$ $O_{P}(1)$. This proves (14). Now, A2 yields (15), since

$$
\begin{aligned}
& \sup _{n \geq 1} E\left[\sup _{\left|v_{1}-v_{2}\right| \leq \delta}\left|d_{1 t n}\left(v_{1}\right)-d_{1 t n}\left(v_{2}\right)\right|\right] \\
\leq & \sup _{n \geq 1}\left[\sup _{\left|v_{1}-v_{2}\right| \leq \delta}\left|\frac{h\left(\widehat{I}_{t-1}, \theta_{0}+n^{-1 / 2} v_{1}\right)-h\left(\widehat{I}_{t-1}, \theta_{0}+n^{-1 / 2} v_{2}\right)}{h\left(I_{t-1}, \theta_{0}\right)}\right|\right] \\
\leq & C \delta .
\end{aligned}
$$

Now, we proceed to verify (16). By Assumption A4 and the Ergodic Theorem

$$
\begin{aligned}
& \sup _{\varepsilon \in(0,1) \cap \mathbb{Q}} n^{-1} \sum_{t=1}^{n} \varepsilon^{-1} \bar{g}_{n, t, K}^{2}\left[\sup _{\left|v_{1}-v_{2}\right| \leq \varepsilon}\left|d_{1 t n}\left(v_{1}\right)-d_{1 t n}\left(v_{2}\right)\right|\right] \\
\leq & C\left\{n^{-1 / 2} \max _{1 \leq t \leq n} E\left[\sup _{\theta \in \Theta_{0}}\left|\frac{\dot{h}\left(\widehat{I}_{t-1}, \theta\right)}{h\left(I_{t-1}, \theta_{0}\right)}\right|\right]\right\} n^{-1} \sum_{t=1}^{n} \bar{g}_{n, t, K}^{2} \\
= & O_{P}(1),
\end{aligned}
$$

Similar conclusions holds for $d_{2 t n}$. Hence, Theorem A1 applies to $\widetilde{K}_{n}(x, v)$. We use this fact to handle $A_{3 n}$ in (18).

Note that for a fixed $(x, v)$, as $n \rightarrow \infty$, our assumptions imply by Cauchy-Schwartz inequality, A4 and $\mathrm{A} 5$, as $n \rightarrow \infty$,

$$
\operatorname{Var}\left(A_{3 n}(x, v)\right) \leq \frac{1}{n} \sum_{t=1}^{n} E\left[\bar{g}_{n, t, K}^{2}\left|F_{u}\left(i_{n, t}(x, v)\right)-F_{u}(x)\right|\right] \rightarrow 0 .
$$

Theorem A1 implies that $A_{1 n}(x, v)$ is asymptotically tight in $\ell^{\infty}(\mathcal{D})$, the Banach space of real-valued bounded functions on $\mathcal{D}$, equipped with the supremum norm $\|z\|_{\mathcal{D}}:=\sup _{g \in \mathcal{D}}|z(g)|$. From the latter fact and the asymptotic tightness of $\widetilde{K}_{n}(x, v)$ and (19), it then follows that

$$
\sup _{\gamma=(x, v) \in \Pi_{K}}\left|A_{3 n}(x, v)\right|=o_{P}(1) .
$$

We proceed to study $A_{2 n}(x, v)$. Define

$$
\begin{aligned}
& \widehat{d}_{1 t n}(v):=\frac{h\left(\widehat{I}_{t-1}, \theta_{0}+n^{-1 / 2} v\right)-h\left(I_{t-1}, \theta_{0}+n^{-1 / 2} v\right)}{h\left(I_{t-1}, \theta_{0}\right)}, \text { and } \\
& \widehat{d}_{2 t n}(v):=\frac{f\left(\widehat{I}_{t-1}, \theta_{0}+n^{-1 / 2} v\right)-f\left(I_{t-1}, \theta_{0}+n^{-1 / 2} v\right)}{h\left(I_{t-1}, \theta_{0}\right)}
\end{aligned}
$$


Note that

$$
\begin{aligned}
A_{2 n}(x, v)= & \frac{1}{\sqrt{n}} \sum_{t=1}^{n} g_{n, t}(v)\left[F_{u}\left(i_{n, t}(x, v)\right)-F_{u}\left(x+x d_{1 t n}(v)\right)\right] \\
& +\frac{1}{\sqrt{n}} \sum_{t=1}^{n} g_{n, t}(v)\left[F_{u}\left(x+x d_{1 t n}(v)\right)-F_{u}(x)\right] \\
: \quad & =B_{1 n}(x, v)+B_{2 n}(x, v) .
\end{aligned}
$$

We write

$$
\begin{aligned}
B_{1 n}(x, v)= & \frac{1}{\sqrt{n}} \sum_{t=1}^{n} g_{n, t}(v) f_{u}\left(\widetilde{i}_{n, t}(x, v)\right) d_{2 t n}(v) \\
= & f_{u}(x) \frac{1}{\sqrt{n}} \sum_{t=1}^{n} g_{n, t}(v) d_{2 t n}(v) \\
& +\frac{1}{\sqrt{n}} \sum_{t=1}^{n} g_{n, t}(v)\left[f_{u}\left(x+x d_{1 t n}(v)\right)-f_{u}(x)\right] d_{2 t n}(v) \\
& +\frac{1}{\sqrt{n}} \sum_{t=1}^{n} g_{n, t}(v)\left[f_{u}\left(\widetilde{i}_{n, t}(x, v)\right)-f_{u}\left(x+x d_{1 t n}(v)\right)\right] d_{2 t n}(v) \\
: \quad & C_{1 n}(x, g, v)+C_{2 n}(x, g, v)+C_{3 n}(x, g, v)
\end{aligned}
$$

for some $\widetilde{i}_{n, t}(x, v)$ between $i_{n, t}(x, v)$ and $x+x d_{1 t n}(v)$.

Assumptions A1, A2, A4 and the Ergodic Theorem imply that for some $\widetilde{\theta}_{n}$ with $\left|\widetilde{\theta}_{n}-\theta_{0}\right| \leq$ $n^{-1 / 2} v$, uniformly in $v$,

$$
\begin{aligned}
\frac{1}{\sqrt{n}} \sum_{t=1}^{n} g_{n, t}(v) d_{2 t n}(v) & =v^{\prime} \frac{1}{n} \sum_{t=1}^{n} g_{n, t}(v) \frac{\dot{f}_{t}\left(\widetilde{\theta}_{n}\right)}{h\left(I_{t-1}, \theta_{0}\right)}+\frac{1}{\sqrt{n}} \sum_{t=1}^{n} g_{n, t}(v) \widehat{d}_{2 t n}(v) \\
& =v^{\prime} E\left[g_{e}\left(I_{t-1}, \theta_{0}\right) \frac{\dot{f}_{t}\left(\theta_{0}\right)}{h\left(I_{t-1}\right)}\right]+o_{P}(1)
\end{aligned}
$$

This is so because

$$
\sup _{v \in \Delta_{K}}\left|\frac{1}{\sqrt{n}} \sum_{t=1}^{n} g_{n, t}(v) \widehat{d}_{2 t n}(v)\right| \leq n^{-1 / 2} \max _{1 \leq t \leq n} \bar{g}_{n, t, K} \sup _{v \in \Delta_{K}}\left|\sum_{t=1}^{n} \widehat{d}_{2 t n}(v)\right|=o_{P}(1),
$$

and, by the uniform law of large numbers of Jennrich (1969),

$$
\sup _{v \in \Delta_{K}}\left|\frac{1}{n} \sum_{t=1}^{n} g_{n, t}(v) \frac{\dot{f}_{t}\left(\widetilde{\theta}_{n}\right)}{h\left(I_{t-1}, \theta_{0}\right)}-E\left[g_{e}\left(I_{t-1}, \theta_{0}\right) \frac{\dot{f}_{t}\left(\theta_{0}\right)}{h\left(I_{t-1}\right)}\right]\right|=o_{P}(1)
$$

Hence,

$$
\sup _{\gamma \in \Pi_{K}}\left|C_{1 n}(x, v)-v^{\prime} E\left[g_{e}\left(I_{t-1}, \theta_{0}\right) \frac{\dot{f}_{t}\left(\theta_{0}\right)}{h\left(I_{t-1}\right)}\right] f_{u}(x)\right|=o_{P}(1)
$$


By the same arguments, A1 and A4 imply

$$
\begin{aligned}
\sup _{\gamma \in \Pi_{K}}\left|C_{2 n}(x, v)\right| \leq & \sup _{\gamma \in \Pi_{K}}\left|v^{\prime} \frac{1}{n} \sum_{t=1}^{n} g_{n, t}(v)\left[f_{u}\left(x+x d_{1 t n}(v)\right)-f_{u}(x)\right] \frac{\dot{f}_{t}\left(\widetilde{\theta}_{n}\right)}{h\left(I_{t-1}, \theta_{0}\right)}\right| \\
& +\sup _{\gamma \in \Pi_{K}}\left|\frac{1}{\sqrt{n}} \sum_{t=1}^{n} g_{n, t}(v)\left[f_{u}\left(x+x d_{1 t n}(v)\right)-f_{u}(x)\right] \widehat{d}_{2 t n}(v)\right| \\
\leq & C K n^{-1 / 2} \max _{1 \leq t \leq n_{\theta \in \Theta_{0}}}\left|\frac{\dot{h}_{t}(\theta)}{h\left(I_{t-1}, \theta_{0}\right)}\right| \sup _{\gamma \in \Pi_{K}}\left|\frac{1}{n} \sum_{t=1}^{n} g_{n, t}(v) \frac{\dot{f}_{t}\left(\widetilde{\theta}_{n}\right)}{h\left(I_{t-1}, \theta_{0}\right)}\right| \\
& +\sup _{\gamma \in \Pi_{K}}\left|\frac{1}{n} \sum_{t=1}^{n} g_{n, t}(v) \widehat{d}_{1 t n}(v) \frac{\dot{f}_{t}\left(\widetilde{\theta}_{n}\right)}{h\left(I_{t-1}, \theta_{0}\right)}\right| \\
& +\sup _{x \in \mathbb{R}}\left|f_{u}(x)\right| \sup _{\gamma \in \Pi_{K}}\left|\frac{1}{\sqrt{n}} \sum_{t=1}^{n} g_{n, t}(v) \widehat{d}_{2 t n}(v)\right| \\
= & o_{P}(1) .
\end{aligned}
$$

Similarly, it can be proved that $\sup _{\gamma \in \Pi_{K}}\left|C_{3 n}(x, g, v)\right|=o_{P}(1)$.

As for $B_{2 n}(x, v)$, by $\mathrm{A} 1$

$$
\begin{aligned}
B_{2 n}(x, v)= & \frac{1}{\sqrt{n}} \sum_{t=1}^{n} g_{n, t}(v) f_{u}\left(x+x \widetilde{d_{1 t n}}(v)\right) x d_{1 t n}(v) \\
= & f_{u}(x) x \frac{1}{\sqrt{n}} \sum_{t=1}^{n} g_{n, t}(v) d_{1 t n}(v) \\
& +\frac{1}{\sqrt{n}} \sum_{t=1}^{n} g_{n, t}(v)\left[x f_{u}\left(x+x \widetilde{d_{1 t n}}(v)\right)-x f_{u}(x)\right] d_{1 t n}(v) \\
: \quad & =D_{1 n}(x, v)+D_{2 n}(x, v)
\end{aligned}
$$

for some $x+x \widetilde{d_{1 t n}}(v)$ between $x+x d_{1 t n}(v)$ and $x$.

Assumptions A1, A2, A4 and the uniform law of large numbers of Jennrich (1969) imply that

$$
\sup _{\gamma \in \Pi_{K}}\left|D_{1 n}(x, g, v)-v^{\prime} E\left[g_{e}\left(I_{t-1}, \theta_{0}\right) \frac{\dot{h}_{t}\left(\theta_{0}\right)}{h\left(I_{t-1}\right)}\right] f_{u}(x) x\right|=o_{P}(1) .
$$

Similar arguments to those for $C_{j n}, j=2,3$, imply $\sup _{\gamma \in \Pi_{K}}\left|D_{2 n}(x, g, v)\right|=o_{P}(1)$.

Therefore, summarizing we have proved for each fixed $K>0$,

$$
L_{1, n}:=\sup _{\gamma \in \Pi_{K}}\left|K_{n}(x, v)-\frac{1}{\sqrt{n}} \sum_{t=1}^{n} g_{n, t}(v)\left[1\left(u_{t} \leq x\right)-F_{u}(x)\right]-v^{\prime} b\left(x, g, \theta_{0}\right)\right|=o_{P}(1) .
$$

To conclude the proof of Theorem 1 note that, denoting $g_{e}\left(\cdot, \theta_{n}\right)=g\left(\cdot, \theta_{n}\right)-E\left[g\left(I_{t-1}, \theta_{0}\right)\right]$,

$$
\begin{aligned}
R_{n, g}^{1}(x)= & \frac{1}{\sqrt{n}} \sum_{t=1}^{n} g_{e}\left(\widehat{I}_{t-1}, \theta_{n}\right)\left\{1\left(\widehat{u}_{t}\left(\theta_{n}\right) \leq x\right)-F_{u}(x)\right\} \\
& \left.+\left[E\left[g\left(I_{t-1}, \theta_{0}\right)\right]\right]-\bar{g}\left(\theta_{n}\right)\right] \frac{1}{\sqrt{n}} \sum_{t=1}^{n}\left\{1\left(\widehat{u}_{t}\left(\theta_{n}\right) \leq x\right)-F_{u}(x\}\right) \\
: \quad & =A_{1 n}(x)+A_{2 n}(x) .
\end{aligned}
$$


It is straightforward to prove that $A_{2 n}(x)=o_{P}(1)$, uniformly in $x \in \mathbb{R}$. Hence, since $\sqrt{n}\left(\theta_{n}-\theta_{0}\right)=$ $O_{P}(1)$, for any $\varepsilon>0$, there exists a $K>0$ such that the event $C_{n, K}=\left\{\sqrt{n}\left|\theta_{n}-\theta_{0}\right|>K\right\}$ satisfies $\operatorname{Pr}\left(C_{n, K}\right)<\varepsilon / 2$ for all $n$ sufficiently large. To simplify notation, define

$$
L_{2, n}:=\sup _{x \in \mathbb{R}}\left|R_{n, g}^{1}(x)-\frac{1}{\sqrt{n}} \sum_{t=1}^{n} g_{e}\left(I_{t-1}, \theta_{n}\right)\left[1\left(u_{t} \leq x\right)-F_{u}(x)\right]-\sqrt{n}\left(\theta_{n}-\theta_{0}\right)^{\prime} b\left(x, g, \theta_{0}\right)\right| .
$$

Thus, because $A_{1 n}(x)=K_{n}\left(x, \sqrt{n}\left(\theta_{n}-\theta_{0}\right)\right)$, we conclude using (20) that, for any $\delta>0$, we can choose $n$ sufficiently large such that

$$
\begin{aligned}
\operatorname{Pr}\left(L_{2, n}>\delta\right) & \leq \operatorname{Pr}\left(L_{2, n}>\delta, \sqrt{n}\left|\theta_{n}-\theta_{0}\right| \leq K\right)+\operatorname{Pr}\left(C_{n, K}\right) \\
& \leq \operatorname{Pr}\left(L_{1, n}>\delta\right)+\operatorname{Pr}\left(C_{n, K}\right) \\
& \leq \varepsilon / 2+\varepsilon / 2=\varepsilon
\end{aligned}
$$

Since $\varepsilon$ and $\delta$ were arbitrary, we conclude that $L_{2, n}=o_{P}(1)$. Finally, Theorem 1 is proved after noticing that, by A5,

$$
\sup _{x \in \mathbb{R}}\left|\frac{1}{\sqrt{n}} \sum_{t=1}^{n}\left[g_{e}\left(I_{t-1}, \theta_{n}\right)-g_{e}\left(I_{t-1}, \theta_{0}\right)\right]\left[1\left(u_{t} \leq x\right)-F_{u}(x)\right]\right|=o_{P}(1) .
$$

To emphasize the dependence of $X_{t}(\theta)$ on $I_{t-1}$, we write when it is convenient $X_{t}(\theta) \equiv X\left(I_{t-1}, \theta\right)$. Proof of Corollary 1: Without loss of generality assume that $\widehat{X}_{t}\left(\widehat{\theta}_{n}\right)$ are centered in the sample (otherwise, write the residual with centered regressors). Write

$$
\begin{aligned}
K_{n, \widehat{g}}^{1}(\alpha)= & \frac{1}{\sqrt{n}} \sum_{t=1}^{n}\left\{g_{0}\left(\widehat{I}_{t-1}\right)-\widehat{\gamma}_{n}^{\prime}\left(\widehat{\theta}_{n}\right) \widehat{X}_{t}\left(\widehat{\theta}_{n}\right)\right\} 1\left(\widehat{u}_{t} \leq x\right) \\
= & \frac{1}{\sqrt{n}} \sum_{t=1}^{n}\left\{g_{0}\left(\widehat{I}_{t-1}\right)-\gamma^{\prime}\left(\theta_{0}\right) \widehat{X}_{t}\left(\widehat{\theta}_{n}\right)\right\} 1\left(\widehat{u}_{t} \leq x\right) \\
& -\left(\widehat{\gamma}_{n}^{\prime}\left(\widehat{\theta}_{n}\right)-\gamma^{\prime}\left(\theta_{0}\right)\right) \frac{1}{\sqrt{n}} \sum_{t=1}^{n} \widehat{X}_{t}\left(\widehat{\theta}_{n}\right) 1\left(\widehat{u}_{t} \leq x\right) \\
: & =I_{1 n}(x)+I_{2 n}(x) .
\end{aligned}
$$

From the uniform law of large numbers of Jennrich (1969) it follows that

$$
\left|\widehat{\gamma}^{\prime}\left(\widehat{\theta}_{n}\right)-\gamma^{\prime}\left(\theta_{0}\right)\right|=o_{P}(1)
$$

and from Theorem A1,

$$
\sup _{x \in \mathbb{R}}\left|\frac{1}{\sqrt{n}} \sum_{t=1}^{n} \widehat{X}_{t}\left(\widehat{\theta}_{n}\right) 1\left(\widehat{u}_{t} \leq x\right)\right|=O_{P}(1) .
$$

Hence $\sup _{x \in \mathbb{R}}\left|I_{2 n}(x)\right|=o_{P}(1)$. 
As for $I_{1 n}(x)$, again by Theorem 1 applied to $g\left(\widehat{I}_{t-1}, \widehat{\theta}_{n}\right)=\left\{g_{0}\left(\widehat{I}_{t-1}\right)-\gamma^{\prime}\left(\theta_{0}\right) \widehat{X}_{t}\left(\widehat{\theta}_{n}\right)\right\}$, we obtain

$$
\sup _{x \in \mathbb{R}}\left|I_{1 n}(x)-\frac{1}{\sqrt{n}} \sum_{t=1}^{n} g\left(I_{t-1}, \theta_{0}\right)\left[1\left(u_{t} \leq x\right)-F_{u}(x)\right]\right|=o_{P}(1),
$$

with $g\left(I_{t-1}, \theta_{0}\right)=g_{0}\left(I_{t-1}\right)-\gamma^{\prime}\left(\theta_{0}\right) X_{t}\left(\theta_{0}\right)$. The corollary follows from Theorem A1. 


\section{REFERENCES}

Bai, J., Ng, S., (2001) A test for conditional symmetry in time series models. Journal of Econometrics 103, 225-258.

Bera, A. K., Higgins, M. L., (1997) Arch and bilinearity as competing models for nonlinear dependence. Journal of Business and Economic Statistics 15, 43-50.

Billingsley, P., (1999) Convergence of Probability Measures. Second Edition. Wiley, New York.

Bollerslev, T., Chou, R. Y., Kroner, K. F., (1992) ARCH modelling in Finance. Journal of Econometrics 52, 5-59.

Chang, N. M. (1990) Weak convergence of a self-consistent estimator of a survival function with doubly censored data. Annals of Statistics 18, 391-404.

Box, G. and Pierce, D. (1970) Distribution of residual autocorrelations in autoregressive integrated moving average time series models. Journal of the American Statistical Association 65, 1509-1527.

Chen, M. and An, H.Z. (1998). A note on the stationarity and the existence of moments of the GARCH model. Statistica Sinica 8, 505-510.

Chen, X., O. Linton, and I. van Keilegom (2003) Estimation of semiparametric models when the criterion function is not smooth. Econometrica 71, 1591-1608.

Delgado, M.A. and Escanciano, J.C. (2007) Nonparametric tests for conditional symmetry in dynamic models. Journal of Econometrics 141, 652-682.

Escanciano, J.C. (2006). Goodness-of-fit tests for linear and non-linear time series models. Journal of the American Statistical Association 101 531-541.

Escanciano, J.C. (2008) Joint and marginal diagnostic tests for conditional mean and variance specifications. Journal of Econometrics 143, 74-87.

Escanciano, J.C. (2009) On the Lack of Power of Omnibus Specification Tests. Econometric Theory 25, 1-33.

Escanciano, J. C. and Velasco, C. (2006). Generalized Spectral Tests for the Martingale Difference Hypothesis. Journal of Econometrics 134, 151-185.

Fan, J. and Yao, Q. (2003) Nonlinear Time Series: Nonparametric and Parametric Methods. Springer-Verlag, New York.

Francq, C. and Zakoïan, J. M. (2004). Maximum likelihood estimation of pure GARCH and ARMA-GARCH. Bernoulli 10, 605-637.

Gao, J. and King, M. (2004) Adaptive testing in continuous-time diffusion models. Econometric Theory 20 844-882.

Hall, P., and Heyde, C. (1980) Martingale Limit Theory and Its Application. Academic Press, New York.

Härdle, W. and Mammen, E. (1993) Comparing nonparametric versus parametric regression fits. Annals of Statistics 21, 1926-1974.

Hidalgo, J., Zaffaroni, P., (2007) A goodness of fit test for $\operatorname{ARCH}(\infty)$. The Journal of Econometrics 141, 835-875.

Hong, Y. and Lee, T. H. (2003) Diagnostic checking for adequacy of nonlinear time series models. Econometric Theory 19 1065-1121.

Jennrich, R.I. (1969) Asymptotic properties of nonlinear least squares estimators. Annals of Mathematical Statistics 40, 633-643.

Koenker, R., Zhao, Q. (1996) Conditional Quantile Estimation and Inference for ARCH Models. 


\section{Econometric Theory 12, 793-814.}

Koul, H.L. (2002). Weighted Empirical Processes in Dynamic Nonlinear Models. 2nd ed. Lecture Notes in Statistics, Vol. 166, Springer.

Koul, H.L. and Ling, S. (2006). Fitting an error distribution in some heteroscedastic time series models. Annals of Statistics 34, 994-1012.

Li, W.K., Ling, S. \& McAleer, M. (2002) A Survey of Recent Theoretical Results for Time Series Models with GARCH errors. Journal of Economic Survey 16, 245-269.

Li, W. K. and Mak, T. K. (1994) On the squared residual autocorrelation in nonlinear time series with conditional heteroskedasticity. Journal of Time Series Analysis 15, 627-636.

Ljung, G.M. and Box, G.E.P. (1978) A measure of lack of fit in time series models. Biometrika 65, 297-303.

Loudon, G. F., Watt, W. H. and Yadav, P. K. (2000) An empirical analysis of alternative parametric ARCH models. Journal of Applied Econometrics, 15, 117 - 136.

Lundbergh, S.T. and Teräsvirta, T. (2002). Evaluating GARCH models. Journal of Econometrics 110, 417-435.

Ngatchou-Wandji, J., (2005) Checking nonlinear heteroscedastic time series models. Journal of Statistical Planning and Inference 133, 33-68.

Robinson, P.M., Zaffaroni, P., (2006) Pseudo-maximum likelihood estimation of $\mathrm{ARCH}(\infty)$ models. The Annals of Statistics 34, 1049-1074.

Shorack, G. and Wellner, J., (1986) Empirical Processes with Applications to Statistics. Wiley: New York.

Straumann, D., (2005) Estimation in Conditionally Heteroscedastic Time Series Models. Lectures notes in Statistics 181, Springer-Verlag, Berling-Heidelberg.

Stute, W., Xu, W.L and Zhu, L.X. (2008) Model diagnosis for parametric regression in high dimensional spaces. Biometrika 95, 451-467.

Tsay, R.S. (2005). Analysis of financial time series, Second edition, Wiley-Intersci., Hoboken, NJ.

Van der Vaart, A.W. and Wellner, J.A. (1996). Weak Convergence and Empirical Processes. Springer, New York.

Wooldrigde (1990) A unified approach to robust, regression-based specification tests. Econometric Theory 6, 17-43. 
TABLE 1

Rejection Frequencies (over 100) for a Gaussian AR(1)-ARCH(1). 5\% Level

\begin{tabular}{cccccccccc}
\hline & \multicolumn{3}{c}{$\mathbf{n = 1 0 0}$} & \multicolumn{3}{c}{$\mathbf{n = 5 0 0}$} & \multicolumn{3}{c}{$\mathbf{n = 1 0 0 0}$} \\
\cline { 2 - 10 }$g_{0}\left(I_{t-1}\right)$ & $C v M_{n}$ & $K S_{n}$ & $W_{n}$ & $C v M_{n}$ & $K S_{n}$ & $W_{n}$ & $C v M_{n}$ & $K S_{n}$ & $W_{n}$ \\
\hline$Y_{t-1}^{2}$ & 16.1 & 12.4 & 13.0 & 87.9 & 76.0 & 59.7 & 97.6 & 94.1 & 68.2 \\
$Y_{t-1}^{3}$ & 15.5 & 4.0 & 3.4 & 25.5 & 9.5 & 3.5 & 30.1 & 13.0 & 4.0 \\
$Y_{t-1} Y_{t-2}$ & 6.6 & 2.7 & 4.3 & 46.3 & 33.7 & 32.4 & 79.5 & 68.9 & 50.6 \\
$\sin \left(Y_{t-1}\right)$ & 12.5 & 6.1 & 4.2 & 14.3 & 9.7 & 5.3 & 16.0 & 10.2 & 8.2 \\
$\cos \left(Y_{t-1}\right)$ & 24.0 & 19.7 & 34.6 & 98.6 & 94.3 & 88.7 & 100.0 & 100.0 & 93.2 \\
$\varepsilon_{t-1}^{2} 1\left(\varepsilon_{t-1} \leq 0\right)$ & 12.9 & 7.6 & 10.7 & 51.2 & 41.3 & 50.7 & 80.4 & 71.8 & 66.0 \\
$\varepsilon_{t-1}^{2}$ & 14.7 & 10.8 & 8.1 & 71.3 & 59.9 & 57.0 & 93.3 & 88.5 & 66.9 \\
\hline
\end{tabular}
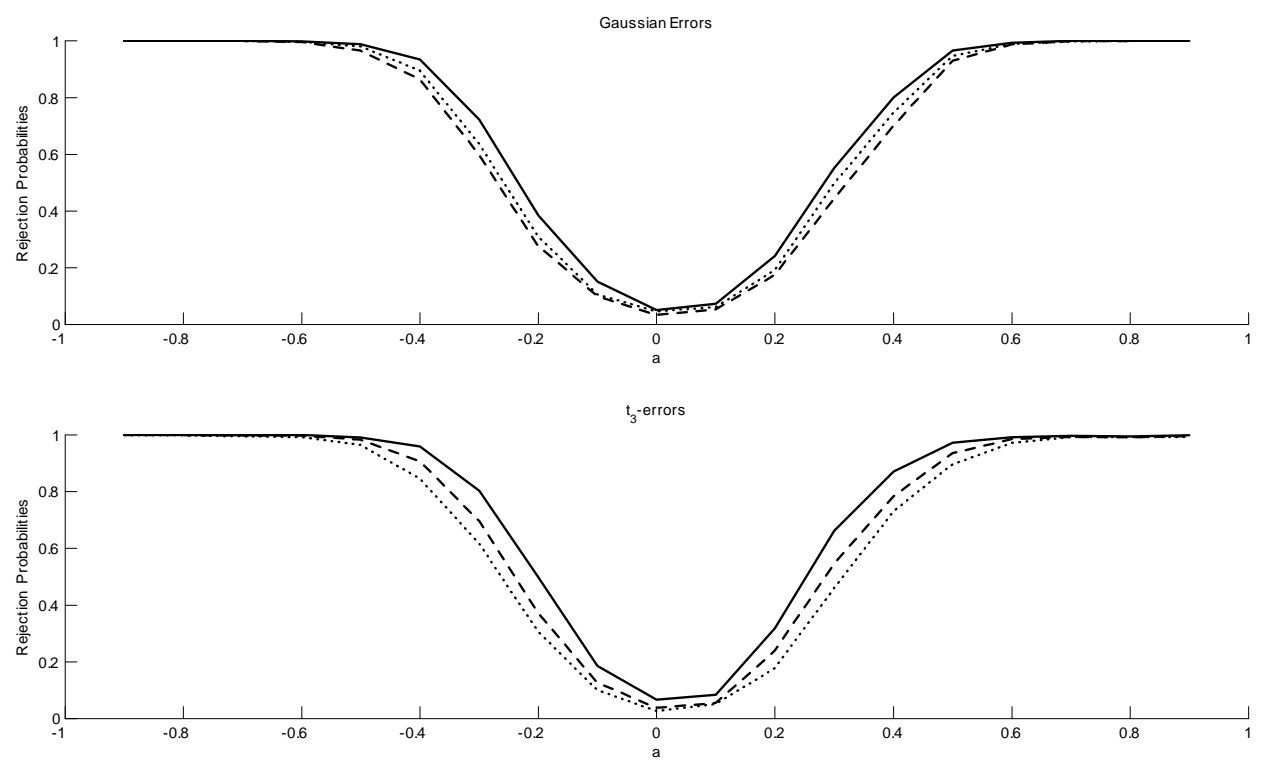

Rejection probabilities for CvM (solid line), KS (dash-dotted line) and W (dotted line) for the AR(1)-GARCH(1,1) model. Sample size $n=100$. Nominal level 5\%. Top panel standard Gaussian innovations. Botton panel Student-t innovations with three degrees of freedom. 
TABle 2

Rejection Frequencies (over 100). 5\% Level

\begin{tabular}{cccccccc}
\hline $\mathbf{n}$ & Test & A0 & ALT1 & ALT2 & ALT3 & ALT4 & ALT5 \\
\hline \hline \multirow{3}{*}{$\mathbf{3 0 0}$} & $C v M_{n}$ & 4.60 & 48.80 & 40.50 & 23.70 & 18.70 & 83.90 \\
& $K S_{n}$ & 3.80 & 38.30 & 35.30 & 21.20 & 16.20 & 84.00 \\
& $W_{n}$ & 4.70 & 44.40 & 31.30 & 19.90 & 16.90 & 63.10 \\
\hline \multirow{3}{*}{$\mathbf{6 0 0}$} & $C v M_{n}$ & 4.90 & 71.20 & 60.90 & 50.00 & 41.30 & 99.40 \\
& $K S_{n}$ & 3.90 & 63.10 & 55.80 & 44.90 & 39.80 & 99.70 \\
& $W_{n}$ & 4.30 & 65.20 & 56.60 & 32.60 & 35.80 & 95.60 \\
\hline \multirow{2}{*}{$\mathbf{1 0 0 0}$} & $K v M_{n}$ & 4.40 & 86.30 & 82.30 & 77.80 & 67.40 & 100.0 \\
& $K S_{n}$ & 3.80 & 77.80 & 77.80 & 71.70 & 65.30 & 100.0 \\
& $W_{n}$ & 4.40 & 83.80 & 81.20 & 54.40 & 59.30 & 99.80 \\
\hline
\end{tabular}

TABLE 3

Estimates of AR(1)-GARCH(1,1) model for the SEP500 daily stock index.

\begin{tabular}{c|c|c|c}
\hline Parameters & $\mathbf{1 9 9 0 - 1 9 9 3}$ & $\mathbf{1 9 9 4 - 1 9 9 7}$ & $\mathbf{1 9 9 8 - 2 0 0 2}$ \\
\hline \hline$a_{0}$ & $0.0257(0.0219)$ & $0.0764(0.0220)$ & $0.0281(0.0370)$ \\
$a_{1}$ & $0.0455(0.0334)$ & $0.0648(0.0355)$ & $0.0200(0.0331)$ \\
$\alpha_{0}$ & $4.7 \mathrm{e}-005(0.0006)$ & $0.0086(0.0033)$ & $0.0808(0.0254)$ \\
$\alpha_{1}$ & $0.0176(0.0033)$ & $0.0772(0.0108)$ & $0.1000(0.0169)$ \\
$\beta_{1}$ & $0.9814(0.0032)$ & $0.9134(0.0146)$ & $0.8567(0.0271)$ \\
\hline
\end{tabular}

Table 4

Model Checks for the SEP500 daily stock index.

\begin{tabular}{|c|c|c|c|c|c|c|c|c|c|}
\hline \multirow[b]{3}{*}{$g_{0}\left(I_{t-1}\right)$} & \multicolumn{3}{|c|}{ 1990-1993 } & \multicolumn{3}{|c|}{ 1994-1997 } & \multicolumn{3}{|c|}{ 1998-2002 } \\
\hline & \multicolumn{3}{|c|}{ GARCH } & \multicolumn{3}{|c|}{ AR-GARCH } & \multicolumn{3}{|c|}{ GARCH } \\
\hline & $C v M_{n}$ & $K S_{n}$ & $W_{n}$ & $C v M_{n}$ & $K S_{n}$ & $W_{n}$ & $C v M_{n}$ & $K S_{n}$ & $W_{n}$ \\
\hline$\left|Y_{t-1}\right|$ & $0.58^{* *}$ & $1.63^{* *}$ & 1.61 & 0.13 & 0.74 & 2.22 & 0.20 & 0.95 & 1.66 \\
\hline$Y_{t-1}^{2}$ & $0.54^{* *}$ & $1.43^{* *}$ & 1.82 & 0.15 & 0.83 & 1.47 & 0.12 & 0.76 & 1.12 \\
\hline$\varepsilon_{t-1}^{2} 1\left(\varepsilon_{t-1} \leq 0\right)$ & 0.11 & 0.76 & 1.01 & 0.29 & 1.03 & 2.82 & $0.40^{*}$ & 1.07 & 0.61 \\
\hline$\left|\varepsilon_{t-1}\right|$ & $0.58^{* *}$ & $1.63^{* *}$ & 1.48 & 0.12 & 0.75 & 2.24 & 0.20 & 0.96 & 1.61 \\
\hline$\varepsilon_{t-1}^{2}$ & $0.54^{* *}$ & $1.44^{* *}$ & 1.62 & 0.15 & 0.82 & 1.34 & 0.12 & 0.76 & 1.06 \\
\hline
\end{tabular}

Note:* Significant at the $10 \%$ level. ${ }^{* *}$ Significant at the $5 \%$ level. Asymptotic tests. 